\title{
DIVERSITY AND STRUCTURE OF THE ICHTHYOLOGIC COMMUNITIES IN THE DIVING SITES IN HOLGUIN (CUBA)
}

\author{
Enrique REYNALDO DE LA CRUZ *, c.a. \\ María Eugenia VEGA CENDEJAS **, Sheila RODRÍGUEZ MACHADO ***, \\ Franklin GARCIA FERNÁNDEZ *** and Antonio VEGA TORRES *
}

\begin{abstract}
* Centro de Investigaciones y Servicios Ambientales de Holguín, Street 18 s/n e/ 1st and Maceo, Delivery, El Llano, Holguín, Cuba, CU-80100, ereynaldodelacruz@gmail.com, vega@cisat.cu (c.a.)

** Centro de Investigación y de Estudios Avanzados del IPN, Unidad Mérida, km 6, antigua carretera a Progreso, Postal mail \#73 Cordemex, Mérida, Yucatán, México, MX-97310, maruvega@cinvestav.mx

*** Instituto de Ciencias del Mar (ICIMAR), Avenue 1st \#18406 e/ 184 y 186, Delivery, Flores, Playa, La Habana, Cuba, CU-11300, sheilaroma89@gmail.com, biolfranklin@gmail.com
\end{abstract}

KEYWORDS: coral reef, marine fish, species richness, equity, replacement.

\section{ABSTRACT}

This study is aimed to determine the diversity and structure of the ichthyologic communities in the coral reefs of Holguín, Cuba. A total of 85 fish species were recorded, including in 32 families and 53 genera. Low species richness and equitability were estimated at different sampling sites throughout the reef system. Cadena de Vita and Canto Chiquito are the sites with the highest number of species 47 and 46 respectively. Cueva 1 and Punta Naranjo were the places with the highest equitability 0.76 . Replacement of fish species among the reef sites studied is poor. Canto Azul with Canto Pionero and La Llanita, sharing 29 species. These results reflect a poor state of conservation of the marine fish communities in Holguín.

RESUMEN: Diversidad y estructura de las comunidades ictiológicas en los arrecifes de coral Holguín (Cuba).

Este estudio pretendió determinar la diversidad y estructura de las comunidades ictiológicas en el arrecife de coral de Holguín, Cuba. Un total de 85 especies fueron registradas, incluidas en 32 familias y 53 géneros. La baja riqueza y equitatividad de especies fueron estimada en los diferentes sitios de muestreo del sistema arrecifal. Cadena de Vita y Canto Chiquito son los sitios con el más alto número de especies 47 y 46 respectivamente. Cueva 1 y Punta Naranjo son los lugares con equitatividad mayor 0.76. El remplazamiento de las especies de peces entre los sitios estudiados del arrecife es pobre. Canto Azul con Canto Pionero y La Llanita, mostraron 29 especies. Este resultado refleja un pobre estado de conservación de las comunidades de peces marinos en Holguín.

REZUMAT: Diversitatea şi structura comunităţilor ihtiologice din recifii de corali Holguin (Cuba).

Acest studiu a avut ca scop determinarea diversităţii şi structurii comunităţilor ihtiologice din recifele de corali din Holguin, Cuba. Au fost înregistrate în total 85 de specii de peşti, incluşi în 32 de familii şi 53 de genuri. O bogăţie în specii şi o echitabilitate reduse au fost estimate în diferite situri de prelevare în sistemul recifal. Cadena de Vita şi Canto Chiquito sunt siturile cu cel mai mare număr de specii 47 şi respectiv 46. Cueva 1 şi Punta Naranjo au fost locurile cu cea mai mare echitabilitate 0.76. Înlocuirea speciilor de peşti printre recifele studiate este redusă. Canto Azul cu Canto Pionero şi La Llanita, împărţind 29 de specii. Aceste rezultate reflect o stare precară de conservare a comunităţilor de peşti marini din Holguín. 


\section{INTRODUCTION}

If more and more world fish stocks were to become systematically overexploited and depleted, then the total marine catch would be expected to decline (Del Monte-Luna and Lluch-Belda, 2016), in this context extensive and intensive fish fauna assessments and monitoring is needed.

The Cuban marine platform is characterized by a wide diversity of marine organisms (Claro, 2006). Fish constitute a group of vital importance in said biota, and the relationship between these and other species of organisms keeps the structure of the different communities stable, which guarantees greater efficiency in the functioning and development of marine ecosystems (González-Sansón and Betancuor, 2003; Caballero et al., 2006). Particularly, in western Cuba, numerous investigations have been carried out related to the issues of structure, composition, and functioning of marine fish communities, as well as other aspects of the ecology of ichthyofauna (Claro and García-Arteaga, 1994; Claro and García-Arteaga, 2001; Chevalier and Cárdenas, 2006). However, in the North Eastern region of Cuba there is little information on the diversity and interaction between marine ichthyologic communities (Reynaldo et al., 2018).

Fish communities show specific organization patterns that can be detected despite the spatial-temporal variability that characterizes them (Ayala-Pérez et al., 2012). Among the emergent properties that characterize them, there are the taxonomic composition, the distribution of their abundances, their diversity (measured as heterogeneity), and equitability, among others (Begon et al., 2006). They also evolve over time and are characterized by their successional stages, within which they maintain structural and functional stability, given by the balance between their components and by environmental constancy (Aviles-Torres et al., 2001). This balance, however, can be affected by external factors, such as anthropic activities, natural environmental changes, or the introduction of species alien to its evolutionary history, known as invasive species (Campos, 2012).

In Cuba, the western portion of the tourist region of Holguín is the most developed area since the 1980s, featuring the highest levels of complexity in the territorial implementation of tourism (La et al., 2012). In this region, the coral reefs display a poor state of conservation, following the proposed methodology by Alcolado and Duran (2011). The investigations carried out by scientific entities in the region have been unpublished rapid inventories within the framework of technical projects, so there is a lack of information on the structure of marine fish communities (Vega et al., 2004). For this reason, it is necessary to determine the diversity and structure of the ichthyologic communities in the coral reefs for tourist use in Holguín, due to the human impact associated pressures, threats and risks.

\section{MATERIAL AND METHODS}

\section{Study area and sampling method}

From the 17th to the 30th of July, 2015, the coastal sector of Guardalavaca beach was sampled, in the northeastern platform of Cuba, northwest of the province of Holguín (Fig. 1). For this, 16 sampling sites (Figs. 1-4) were selected in the front area of the reef at an average depth of $12 \mathrm{~m}$. Each site was georeferenced with a global positioning system, Garmin X12 GPS. Visual censuses were carried out at each site (Brock, 1954) in six linear transects of $50 \mathrm{~m}$ long by two $\mathrm{m}$ wide, occupying a total area of $600 \mathrm{~m}^{2}$. The species of fish observed and the number of individuals belonging to each of them were recorded. Fish species were identified based on descriptions of Humann and Deloach (2002). 


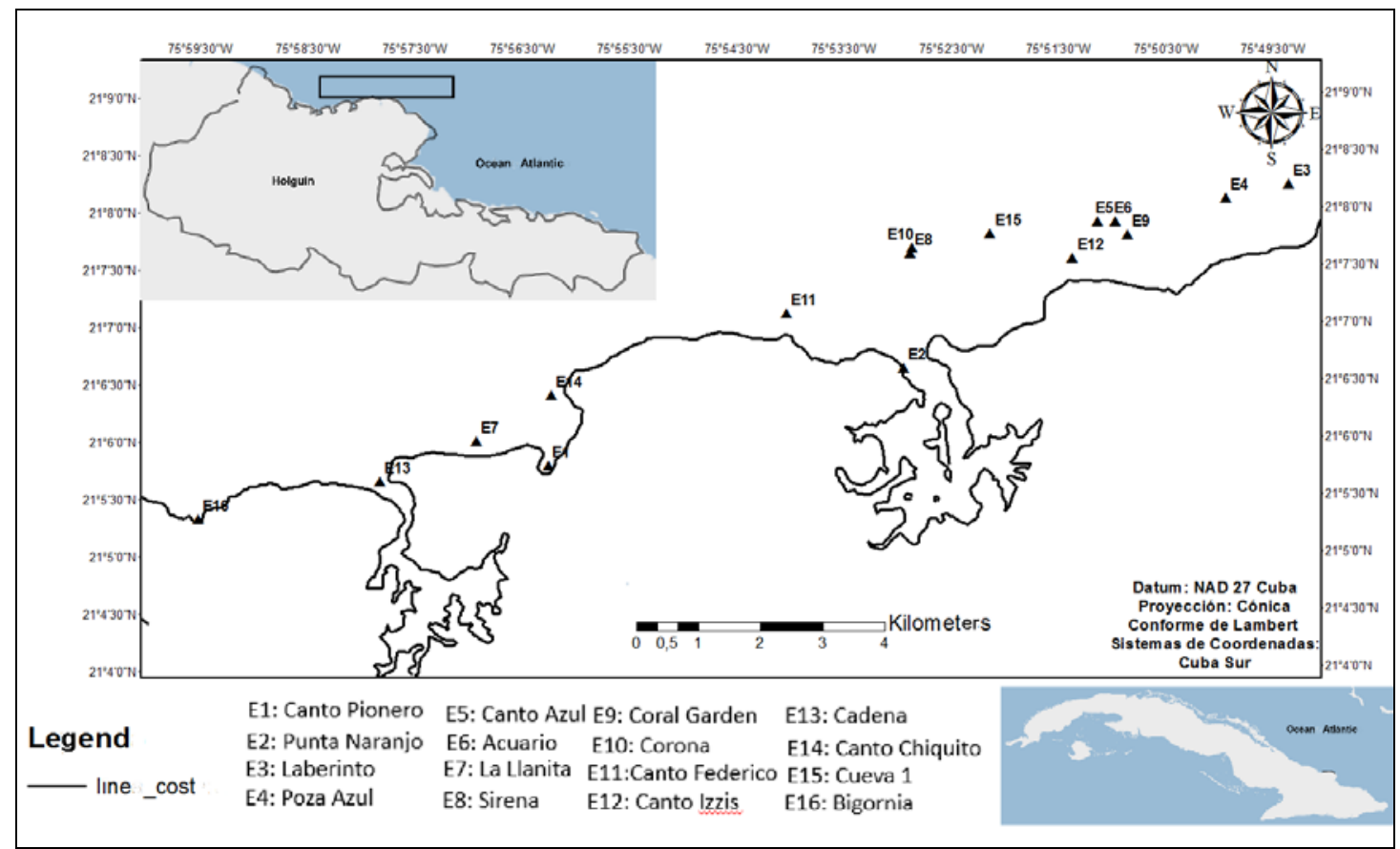

Figure 1: Location of sampling sites.

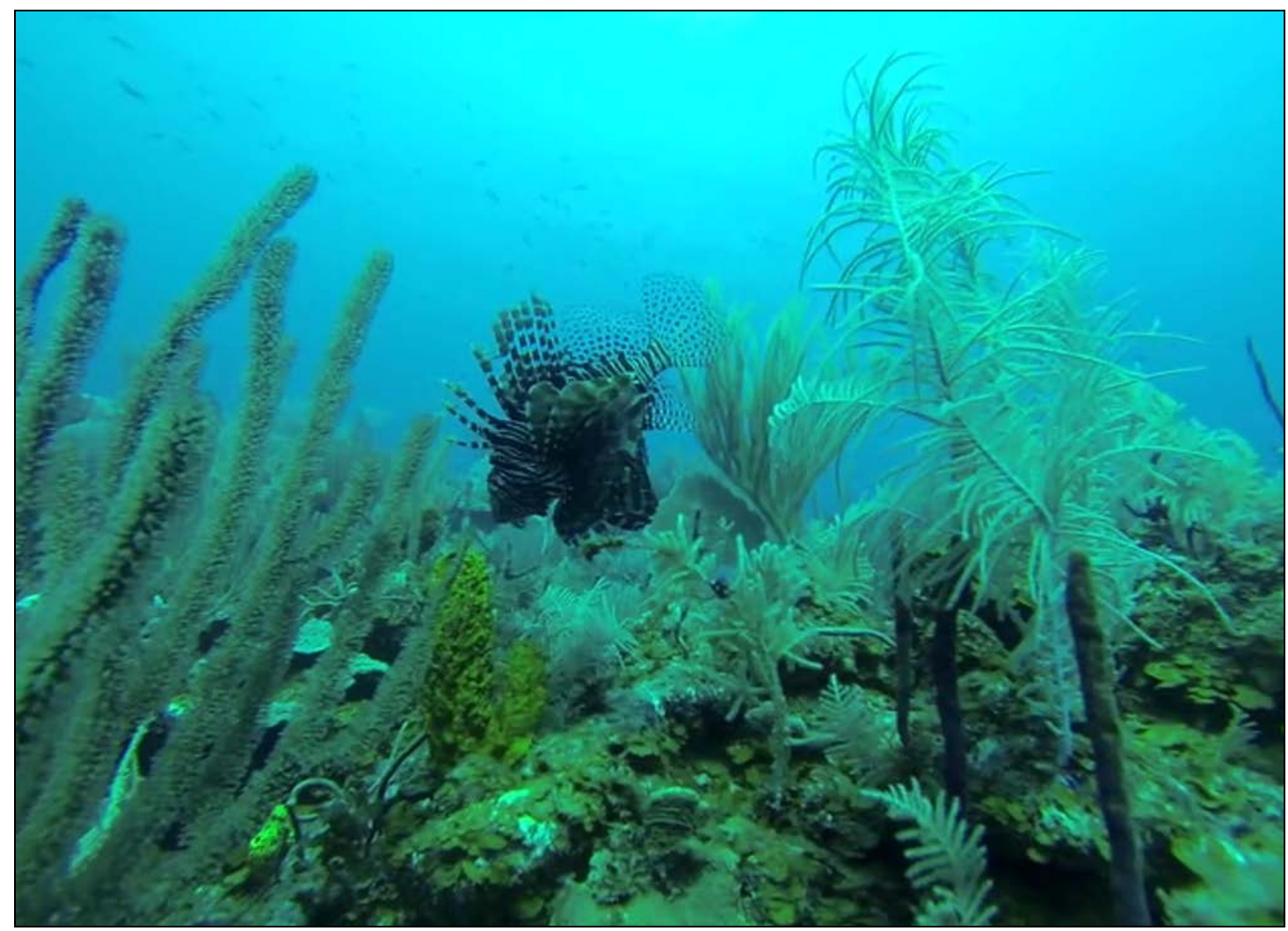

Figure 2: Sampling site. 


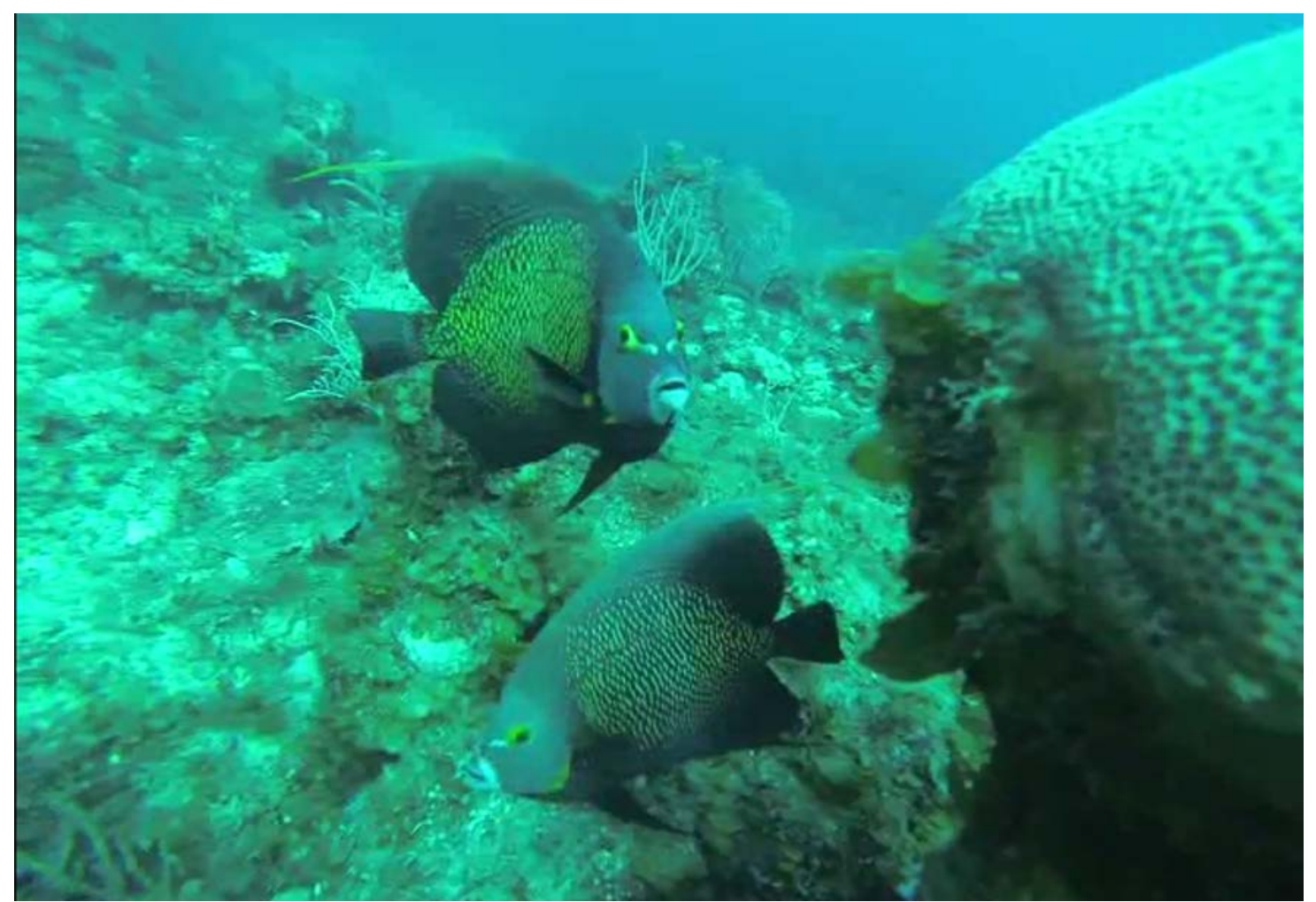

Figure 3: Sampling site.

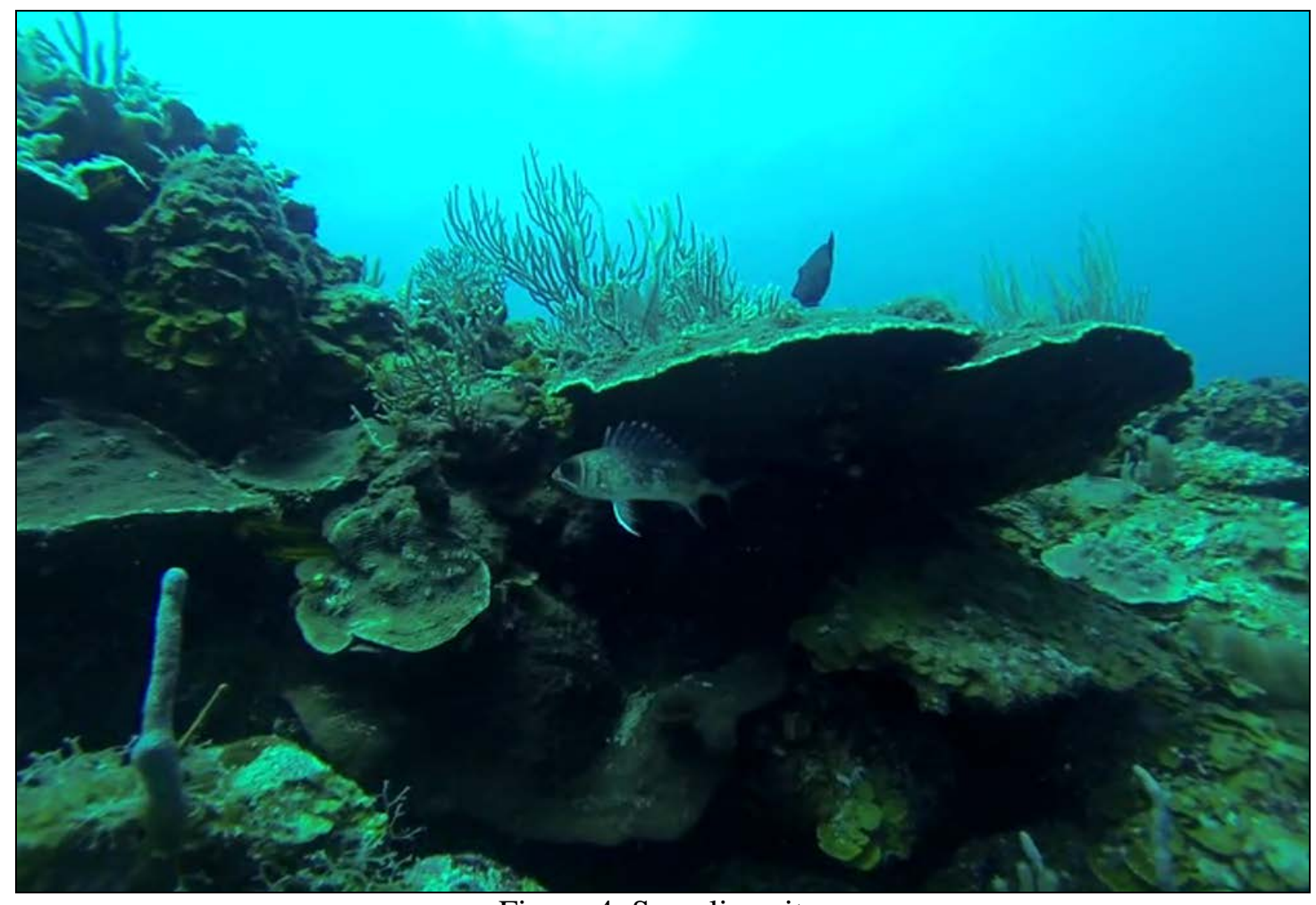

Figure 4: Sampling site. 


\section{Data analysis}

The richness of ichthyologic species was estimated by the number of species recorded (S). The equity of Pielou (J) measures the proportion of the diversity observed in relation to the maximum diversity expected. The dominance of the species (D) was determined following Magurran (2004). Diversity was estimated from Fisher's alpha index, which is based on a logarithmic model of the distribution of the abundance of the species (Magurran, 1988). Beta diversity or diversity between habitats was estimated by the Whittaker index, which measures the degree of species replacement or biotic change through environmental gradients (Whittaker, 1972). Gamma diversity was obtained following the criteria (Landen, 1996), gamma diversity = average alpha + beta, from the richness of species:

Beta calculation based on species richness (Moreno, 2001):

$$
\text { Beta }=\sum_{j} q j(S T-S j)(1)
$$

where:

Beta = Beta diversity;

$\mathrm{qj}=$ Proportional weight of community $j$, based on its area or any other measure of relative importance;

$\mathrm{ST}=$ Total number of species registered in the set of communities;

$\mathrm{Sj}=$ Number of species registered in the community $j$.

For all diversity analyses, bootstrap processing was performed for an $\mathrm{N}=10000$ of the centered type for a 95\% confidence interval. The statistical comparison of the ecological indices between sites was carried out using the diversity permutation test, for 9999 randomized matrices (Brower et al., 1997). The roughness of the seabed $(\mathrm{cm})$ was determined following the AGRRA methodology (2000).

Clustering analysis was used to represent the present fish associations, using the Morisita (1959) index as modified by Horn (1966) as a measure of similarity. This specific analysis constitutes one of the most widely used models to quantify the similarity between communities from quantitative data. Of the quantitative models, it has been suggested that it is the most satisfactory (Magurran, 1988). To determine the statistical differences between the groups ( $70 \%$ similarity), an analysis of similarities (ANOSIM) was performed using 10000 permutations, and a SIMPER to establish the contribution of the abundance of the species, which most influenced the dissimilarity between the groups (Clarke, 1993). The statistical package used was Past. 4.0 (Hammer et al., 2001).

\section{RESULTS}

A total of 85 fish species were recorded, included in 32 families and 53 genera. The most representative species according to their abundances throughout the ecosystem are Clepticus parrae 5,273 individuals, Chromis cyanea 3,202 individuals and Thalassoma bifasciatum 1,014 individuals (Tabs. 2a and 2b).

The ichthyofauna of the Holguín coral reef area present a low species richness (Fig. 2) and equitability (Fig. 3) with an average of 37 species and 0.60 of equitability for 16 studied sites. 


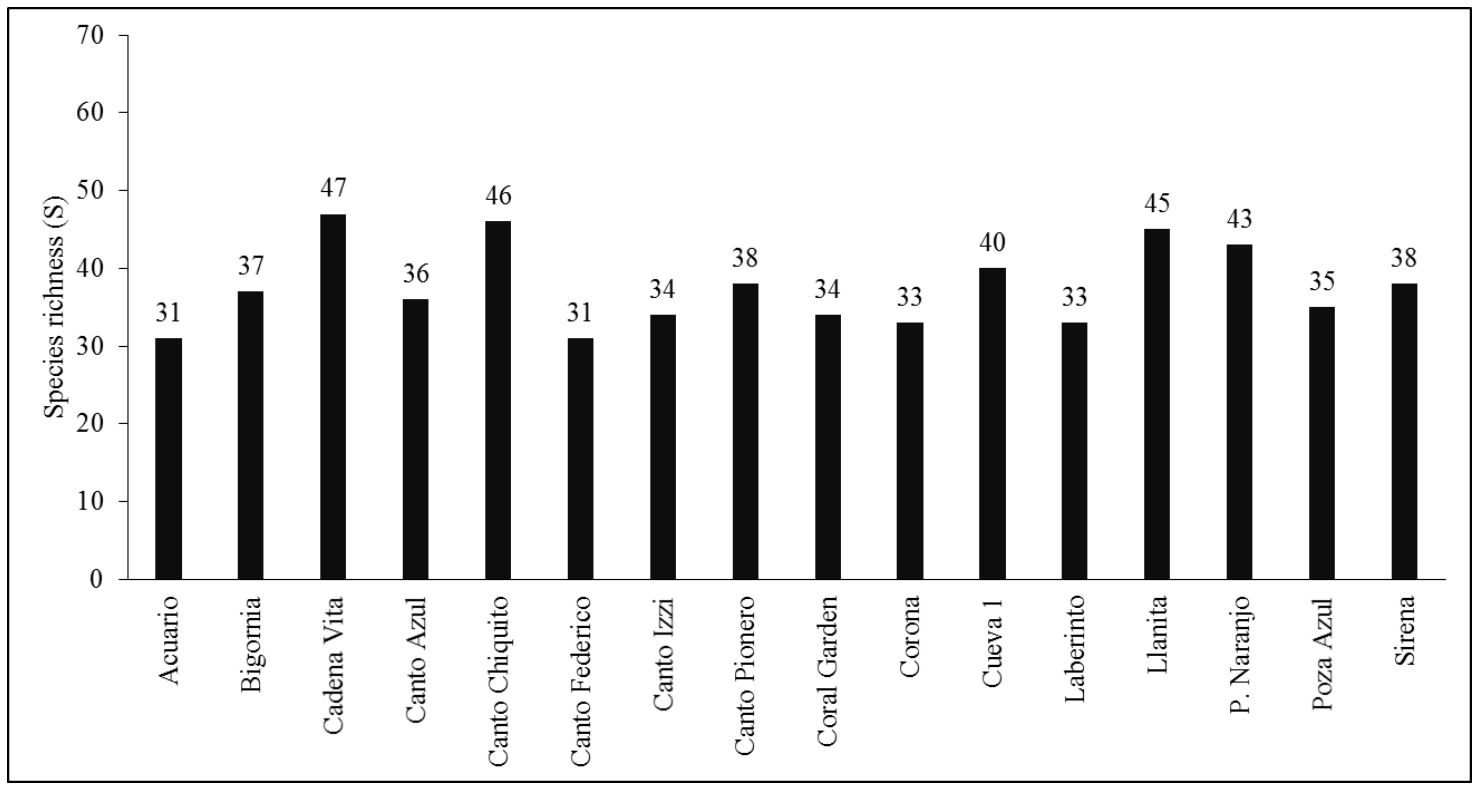

Figure 2: Species richness (S) by dive sites.

Table 1: Ecological indices at Holguín dive sites. The largest values are highlighted in bold. Richness of species Taxa (S), Equity (J), Dominance (D), and Fisher (A).

\begin{tabular}{|c|c|c|c|c|c|c|c|c|c|c|c|c|c|c|c|c|}
\hline 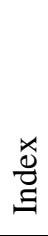 & $\begin{array}{l}\text {. } \\
\text { 忢 } \\
\text { 岁 }\end{array}$ & 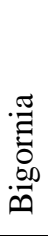 & $\begin{array}{l}\frac{\pi}{\tilde{\Xi}} \\
\frac{\pi}{0}\end{array}$ & 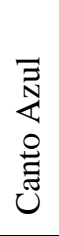 & 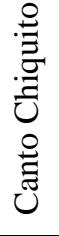 & 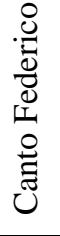 & 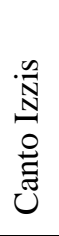 & 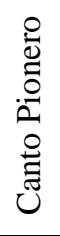 & 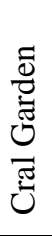 & 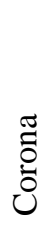 & $\begin{array}{l}\vec{\pi} \\
\stackrel{\pi}{ \pm} \\
0\end{array}$ & 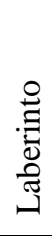 & 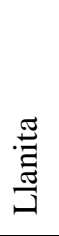 & 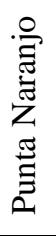 & $\begin{array}{l}\bar{Z} \\
\mathbb{N} \\
\widetilde{N} \\
0 \\
0\end{array}$ & 苛 \\
\hline$S$ & $\vec{m}$ & $\hat{m}$ & f & pి & $\mathscr{q}$ & $\vec{m}$ & ले & $\stackrel{\infty}{m}$ & फे & m & q & $m$ & 字 & $\mathscr{\vartheta}$ & $\stackrel{\text { ㅁ }}{ }$ & 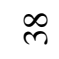 \\
\hline $\mathrm{J}$ & $\stackrel{+}{\stackrel{*}{\circ}}$ & $\begin{array}{l}\mathscr{m} \\
\text { m. }\end{array}$ & 菅 & $\stackrel{M}{\stackrel{M}{0}}$ & ปั & $\begin{array}{l}\stackrel{R}{0} \\
0\end{array}$ & $\stackrel{\infty}{+}$ & $\stackrel{\infty}{+}$ & $\begin{array}{l}\stackrel{L}{\complement n} \\
0\end{array}$ & 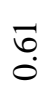 & \begin{tabular}{l}
$\stackrel{0}{0}$ \\
\multirow{0}{*}{}
\end{tabular} & $\begin{array}{l}\text { L } \\
\stackrel{2}{0} \\
0\end{array}$ & :̊. & 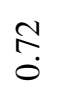 & $\stackrel{0}{0}$ & $\stackrel{L}{0}$ \\
\hline D & $\vec{\sigma}$ & $\stackrel{\infty}{\stackrel{\infty}{0}}$ & $\stackrel{9}{\stackrel{0}{0}}$ & ैㅜㅇ & 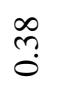 & $\stackrel{\text { Ln }}{\overparen{0}}$ & 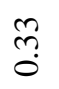 & $\overrightarrow{0}$ & 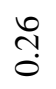 & $\stackrel{\vec{\jmath}}{0}$ & $\stackrel{8}{\circ}$ & 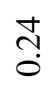 & 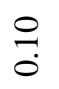 & $\overrightarrow{7}$ & $\overrightarrow{7}$ & $\stackrel{\stackrel{n}{0}}{\circ}$ \\
\hline A & 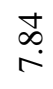 & นึֶ & $\begin{array}{c}\text { m} \\
\infty \\
\infty\end{array}$ & $\begin{array}{l}\text { Oे } \\
\infty\end{array}$ & $\begin{array}{l}\stackrel{\Omega}{\alpha} \\
\infty\end{array}$ & fั & $\stackrel{\text { L̊ }}{\stackrel{2}{人}}$ & $\begin{array}{l}\infty \\
0 \\
0\end{array}$ & $\begin{array}{l}\stackrel{\infty}{\hat{\varphi}} \\
\hat{0}\end{array}$ & बूे & $\stackrel{\sigma}{\varrho}$ & $\stackrel{8}{\stackrel{0}{\circ}}$ & $\begin{array}{l}\infty \\
\stackrel{\infty}{0} \\
\oplus\end{array}$ & $\stackrel{\text { ִֻ }}{=}$ & $\stackrel{゚}{\stackrel{2}{\curvearrowright}}$ & $\begin{array}{l}8 \\
\infty \\
\infty\end{array}$ \\
\hline
\end{tabular}

The sites with the highest species richness are shown in figure 2, where the following stand out: Cadena de Vita and Canto Chiquito with 47 and 46 species, respectively (Tab. 1). In relation to equity, the highest values were obtained in: Cueva 1 and Poza Azul (0.76), followed by Acuario (0.74), Canto Azul (0.73), and Punta Naranjo (0.72) (Tab. 1). The abundance of the species throughout the reef system is low, highlighting C. cyanea 571 ind./100 $\mathrm{m}^{2}$ and C. parrae 1,219 ind./100 $\mathrm{m}^{2}$, as the only species with high abundance, not observing the presence of large groupers or species of fish of bigger size (Tabs. 2a and 2b). 
Table 2a: Abundance of the fish species for each sampling site in $100 \mathrm{~m}^{2}$.

\begin{tabular}{|c|c|c|c|c|c|c|c|c|}
\hline Species & 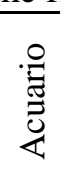 & 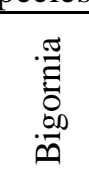 & 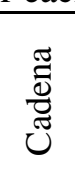 & 总 & 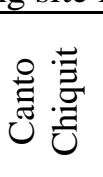 & 号 & 总 & $\begin{array}{l}\text { 总 } \\
\text { 荧 }\end{array}$ \\
\hline Abadeduf saxsatilis & 0 & 0 & 0 & 0 & 16 & 0 & 0 & 16 \\
\hline Acanthostracion polygonia & 1 & 0 & 0 & 0 & 0 & 0 & 0 & 0 \\
\hline Acanthostracion quadricornis & 0 & 0 & 0 & 1 & 0 & 0 & 0 & 0 \\
\hline Acanthurus bahianus & 23 & 21 & 18 & 5 & 5 & 10 & 9 & 0 \\
\hline Acanthurus chirurgus & 0 & 0 & 0 & 0 & 0 & 0 & 0 & 0 \\
\hline Acanthurus coeruleus & 19 & 9 & 9 & 15 & 12 & 9 & 13 & 30 \\
\hline Amblycirrhitus pinos & 0 & 0 & 0 & 0 & 0 & 0 & 0 & 0 \\
\hline Anisotremus virginicus & 0 & 1 & 0 & 5 & 2 & 1 & 0 & 4 \\
\hline Aulostomus macalatus & 0 & 0 & 3 & 0 & 3 & 0 & 0 & 3 \\
\hline Bodianus rufus & 0 & 3 & 3 & 1 & 1 & 0 & 1 & 1 \\
\hline Calamus calamus & 0 & 0 & 0 & 0 & 0 & 0 & 0 & 0 \\
\hline Cantherhines macrocerus & 1 & 0 & 0 & 0 & 0 & 0 & 0 & 0 \\
\hline Cantherhines pullus & 0 & 1 & 0 & 0 & 0 & 0 & 0 & 0 \\
\hline Canthigaster rostrata & 3 & 1 & 10 & 0 & 4 & 1 & 0 & 7 \\
\hline Caranx ruber & 0 & 2 & 0 & 0 & 2 & 0 & 0 & 0 \\
\hline Cephalopholis cruentata & 1 & 4 & 6 & 1 & 3 & 1 & 4 & 4 \\
\hline Cephalopholis fulva & 10 & 8 & 1 & 1 & 4 & 8 & 5 & 4 \\
\hline Chaetodon capistratus & 2 & 4 & 12 & 14 & 7 & 4 & 11 & 8 \\
\hline Chaetodon ocellatus & 0 & 6 & 2 & 0 & 0 & 0 & 1 & 0 \\
\hline Chaetodon sedentarius & 0 & 0 & 1 & 0 & 0 & 0 & 0 & 0 \\
\hline Chaetodon striatus & 0 & 0 & 0 & 0 & 0 & 0 & 0 & 0 \\
\hline Chromis cyanea & 96 & 269 & 571 & 148 & 350 & 24 & 467 & 487 \\
\hline Chromis insolata & 0 & 0 & 0 & 1 & 0 & 0 & 0 & 0 \\
\hline Clepticus parrae & 0 & 1219 & 701 & 14 & 947 & 104 & 0 & 813 \\
\hline Coryphopterus personatus & 0 & 0 & 15 & 30 & 22 & 0 & 3 & 41 \\
\hline Epinephelus flavolimbatus & 0 & 0 & 0 & 0 & 0 & 0 & 0 & 0 \\
\hline Epinephelus guttatus & 0 & 0 & 0 & 0 & 0 & 0 & 3 & 0 \\
\hline Equetus punctatus & 0 & 0 & 0 & 0 & 1 & 0 & 0 & 0 \\
\hline Gramma loreto & 0 & 0 & 2 & 17 & 16 & 0 & 6 & 16 \\
\hline Gramma melacara & 0 & 0 & 0 & 0 & 0 & 0 & 0 & 0 \\
\hline Haemulon aurolineatum & 0 & 0 & 345 & 0 & 1 & 0 & 0 & 0 \\
\hline Haemulon bonariense & 0 & 0 & 0 & 0 & 0 & 0 & 1 & 0 \\
\hline Haemulon carbonarium & 0 & 0 & 0 & 0 & 0 & 0 & 0 & 0 \\
\hline Haemulon flavolineatum & 4 & 31 & 187 & 13 & 10 & 17 & 5 & 28 \\
\hline Haemulon macrostomum & 0 & 0 & 0 & 1 & 0 & 0 & 0 & 2 \\
\hline Haemulon plumieri & 1 & 0 & 19 & 2 & 1 & 2 & 1 & 1 \\
\hline Haemulon sciurus & 2 & 0 & 1 & 1 & 3 & 16 & 0 & 11 \\
\hline Halichoeres bivittatus & 0 & 0 & 0 & 0 & 0 & 0 & 0 & 0 \\
\hline Halichoeres garnoti & 10 & 9 & 4 & 2 & 7 & 10 & 13 & 2 \\
\hline Halichoeres maculipinna & 0 & 0 & 0 & 0 & 0 & 0 & 0 & 0 \\
\hline Holacanthus ciliaris & 0 & 0 & 0 & 0 & 1 & 0 & 0 & 0 \\
\hline
\end{tabular}


Table 2a (continued): Abundance of the fish species for each sampling site in $100 \mathrm{~m}^{2}$.

\begin{tabular}{|c|c|c|c|c|c|c|c|c|}
\hline Holacanthus tricolor & 2 & 5 & 12 & 3 & 1 & 1 & 4 & 1 \\
\hline Holocentrus adscensionis & 0 & 1 & 2 & 0 & 0 & 1 & 0 & 0 \\
\hline Holocentrus rufus & 2 & 0 & 21 & 5 & 5 & 3 & 3 & 22 \\
\hline Hypoplectrus gummigutta & 0 & 0 & 1 & 0 & 1 & 1 & 0 & 0 \\
\hline Hypoplectrus guttavarium & 0 & 0 & 1 & 1 & 1 & 0 & 0 & 1 \\
\hline Hypoplectrus indigo & 0 & 0 & 2 & 0 & 0 & 0 & 1 & 1 \\
\hline Hypoplectrus nigricans & 0 & 0 & 1 & 0 & 0 & 0 & 0 & 0 \\
\hline Hypoplectrus puella & 0 & 4 & 8 & 1 & 8 & 1 & 7 & 4 \\
\hline Lactophrys triqueter & 1 & 1 & 0 & 0 & 2 & 2 & 1 & 0 \\
\hline Lutjanus analis & 0 & 0 & 0 & 0 & 0 & 3 & 0 & 0 \\
\hline Lutjanus apodus & 4 & 2 & 2 & 8 & 0 & 0 & 1 & 1 \\
\hline Lutjanus griseus & 0 & 0 & 0 & 0 & 0 & 0 & 0 & 0 \\
\hline Lutjanus jocu & 0 & 0 & 0 & 0 & 1 & 0 & 0 & 0 \\
\hline Lutjanus mahogoni & 0 & 0 & 3 & 11 & 1 & 0 & 0 & 32 \\
\hline Lutjanus synagris & 0 & 0 & 0 & 0 & 0 & 0 & 0 & 0 \\
\hline Microspathodon chrysurus & 2 & 0 & 0 & 2 & 0 & 0 & 0 & 2 \\
\hline Mulloidichthys martinicus & 0 & 3 & 12 & 2 & 2 & 0 & 3 & 8 \\
\hline Mycteroperca bonaci & 0 & 0 & 0 & 0 & 1 & 0 & 0 & 0 \\
\hline Myripristis jacobus & 27 & 0 & 0 & 9 & 0 & 0 & 0 & 0 \\
\hline Neoniphon marianus & 0 & 3 & 3 & 0 & 2 & 0 & 0 & 0 \\
\hline Ocyurus chrysurus & 0 & 0 & 3 & 2 & 8 & 13 & 0 & 3 \\
\hline Pomacanthus arcuatus & 0 & 1 & 3 & 0 & 0 & 0 & 0 & 0 \\
\hline Pomacanthus paru & 0 & 0 & 0 & 0 & 0 & 0 & 0 & 0 \\
\hline Prognathodes aculeatus & 0 & 1 & 1 & 1 & 0 & 3 & 1 & 0 \\
\hline Pseudopeneus maculatus & 0 & 1 & 1 & 0 & 3 & 0 & 0 & 0 \\
\hline Pterois volitans & 0 & 1 & 0 & 0 & 6 & 0 & 0 & 0 \\
\hline Sargocentron vexillarium & 0 & 0 & 0 & 0 & 0 & 0 & 0 & 0 \\
\hline Scarus iserti & 7 & 53 & 116 & 26 & 31 & 10 & 9 & 57 \\
\hline Scarus taeniopterus & 75 & 43 & 74 & 41 & 68 & 27 & 71 & 5 \\
\hline Scarus vetula & 2 & 0 & 1 & 1 & 1 & 0 & 0 & 0 \\
\hline Serranus tigrinus & 0 & 3 & 0 & 0 & 0 & 5 & 1 & 0 \\
\hline Sparisoma atomarium & 0 & 2 & 14 & 0 & 16 & 0 & 1 & 0 \\
\hline Sparisoma aurofrenatum & 25 & 21 & 24 & 28 & 23 & 24 & 39 & 18 \\
\hline Sparisoma chrysopterum & 0 & 0 & 0 & 0 & 0 & 0 & 0 & 0 \\
\hline Sparisoma rubripinne & 1 & 0 & 0 & 0 & 0 & 0 & 0 & 0 \\
\hline Sparisoma viride & 7 & 3 & 7 & 1 & 3 & 3 & 6 & 20 \\
\hline Stegastes adustus & 1 & 0 & 0 & 0 & 0 & 0 & 0 & 0 \\
\hline Stegastes diencaeus & 28 & 6 & 2 & 0 & 3 & 1 & 5 & 13 \\
\hline Stegastes leucostictus & 1 & 0 & 0 & 0 & 0 & 0 & 0 & 2 \\
\hline Stegastes partitus & 23 & 4 & 6 & 30 & 0 & 17 & 5 & 1 \\
\hline Stegastes planifrons & 3 & 5 & 6 & 0 & 6 & 0 & 1 & 4 \\
\hline Stegastes variabilis & 0 & 2 & 7 & 0 & 9 & 1 & 2 & 2 \\
\hline Synodus synodus & 0 & 0 & 1 & 0 & 0 & 0 & 0 & 0 \\
\hline Thalassoma bifasciatum & 17 & 37 & 17 & 49 & 13 & 143 & 161 & 20 \\
\hline Average abundance & 4.71 & 21.05 & 26.6 & 5.8 & 19.21 & 5.48 & 10.17 & 19.94 \\
\hline Total species number & 31 & 37 & 47 & 36 & 46 & 31 & 34 & 38 \\
\hline
\end{tabular}


Table 2b: Abundance of the fish species for each sampling site in $100 \mathrm{~m}^{2}$.

\begin{tabular}{|c|c|c|c|c|c|c|c|c|}
\hline Species & 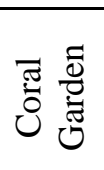 & 苂 & Ũ & 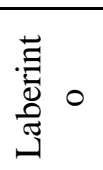 & 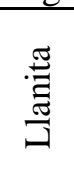 & 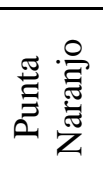 & $\begin{array}{l}\vec{Z} \\
\stackrel{N}{N} \\
\text { N } \\
\text { D. }\end{array}$ & $\stackrel{\overparen{D}}{:}$ \\
\hline Abadeduf saxsatilis & 2 & 0 & 0 & 0 & 0 & 10 & 0 & 0 \\
\hline Acanthostracion polygonia & 0 & 0 & 0 & 0 & 0 & 0 & 0 & 0 \\
\hline Acanthostracion quadricornis & 0 & 0 & 0 & 0 & 0 & 0 & 0 & 0 \\
\hline Acanthurus bahianus & 15 & 4 & 1 & 5 & 7 & 3 & 14 & 7 \\
\hline Acanthurus chirurgus & 1 & 0 & 0 & 0 & 0 & 22 & 0 & 0 \\
\hline Acanthurus coeruleus & 16 & 27 & 22 & 7 & 24 & 0 & 11 & 24 \\
\hline Amblycirrhitus pinos & 0 & 0 & 0 & 0 & 0 & 0 & 0 & 1 \\
\hline Anisotremus virginicus & 0 & 6 & 5 & 300 & 1 & 6 & 0 & 0 \\
\hline Aulostomus macalatus & 0 & 0 & 1 & 0 & 0 & 0 & 1 & 0 \\
\hline Bodianus rufus & 3 & 0 & 0 & 0 & 3 & 0 & 0 & 2 \\
\hline Calamus calamus & 0 & 0 & 0 & 1 & 0 & 0 & 0 & 0 \\
\hline Cantherhines macrocerus & 0 & 0 & 0 & 0 & 0 & 0 & 0 & 0 \\
\hline Cantherhines pullus & 0 & 0 & 0 & 0 & 0 & 0 & 0 & 0 \\
\hline Canthigaster rostrata & 3 & 0 & 1 & 0 & 2 & 0 & 0 & 0 \\
\hline Caranx ruber & 0 & 2 & 0 & 1 & 1 & 2 & 2 & 1 \\
\hline Cephalopholis cruentata & 2 & 1 & 2 & 1 & 7 & 1 & 1 & 0 \\
\hline Cephalopholis fulva & 12 & 0 & 2 & 1 & 15 & 3 & 5 & 0 \\
\hline Chaetodon capistratus & 3 & 11 & 1 & 5 & 8 & 5 & 8 & 13 \\
\hline Chaetodon ocellatus & 0 & 0 & 0 & 0 & 0 & 2 & 1 & 3 \\
\hline Chaetodon sedentarius & 0 & 0 & 0 & 0 & 0 & 0 & 0 & 0 \\
\hline Chaetodon striatus & 0 & 3 & 0 & 0 & 0 & 0 & 0 & 0 \\
\hline Chromis cyanea & 117 & 132 & 80 & 63 & 155 & 60 & 116 & 67 \\
\hline Chromis insolata & 0 & 0 & 1 & 0 & 0 & 0 & 0 & 0 \\
\hline Clepticus parrae & 470 & 317 & 20 & 33 & 119 & 133 & 183 & 200 \\
\hline Coryphopterus personatus & 0 & 0 & 10 & 0 & 106 & 30 & 0 & 0 \\
\hline Epinephelus flavolimbatus & 0 & 0 & 0 & 0 & 3 & 0 & 0 & 0 \\
\hline Epinephelus guttatus & 0 & 0 & 0 & 0 & 0 & 0 & 0 & 0 \\
\hline Equetus punctatus & 0 & 0 & 0 & 0 & 1 & 0 & 0 & 0 \\
\hline Gramma loreto & 0 & 12 & 15 & 0 & 8 & 9 & 0 & 1 \\
\hline Gramma melacara & 0 & 2 & 0 & 0 & 0 & 0 & 0 & 0 \\
\hline Haemulon aurolineatum & 0 & 0 & 0 & 0 & 0 & 0 & 0 & 0 \\
\hline Haemulon bonariense & 0 & 0 & 0 & 0 & 0 & 0 & 0 & 0 \\
\hline Haemulon carbonarium & 0 & 0 & 0 & 201 & 0 & 0 & 4 & 0 \\
\hline Haemulon flavolineatum & 2 & 6 & 40 & 0 & 13 & 2 & 13 & 4 \\
\hline Haemulon macrostomum & 0 & 0 & 0 & 1 & 22 & 0 & 0 & 2 \\
\hline Haemulon plumieri & 0 & 0 & 2 & 0 & 3 & 0 & 6 & 2 \\
\hline Haemulon sciurus & 2 & 3 & 3 & 0 & 3 & 2 & 8 & 1 \\
\hline Halichoeres bivittatus & 0 & 0 & 0 & 0 & 0 & 12 & 7 & 0 \\
\hline Halichoeres garnoti & 14 & 4 & 10 & 7 & 3 & 5 & 14 & 1 \\
\hline Halichoeres maculipinna & 1 & 0 & 0 & 0 & 0 & 0 & 0 & 0 \\
\hline Holacanthus ciliaris & 0 & 0 & 1 & 0 & 0 & 0 & 0 & 1 \\
\hline
\end{tabular}


Table 2b (continued): Abundance of the fish species for each sampling site in $100 \mathrm{~m}^{2}$.

\begin{tabular}{|c|c|c|c|c|c|c|c|c|}
\hline Holacanthus tricolor & 3 & 2 & 2 & 4 & 3 & 2 & 6 & 2 \\
\hline Holocentrus adscensionis & 1 & 0 & 1 & 2 & 1 & 0 & 9 & 1 \\
\hline Holocentrus rufus & 0 & 3 & 9 & 0 & 5 & 2 & 16 & 3 \\
\hline Hypoplectrus gummigutta & 2 & 0 & 0 & 0 & 0 & 0 & 0 & 0 \\
\hline Hypoplectrus guttavarium & 0 & 0 & 0 & 0 & 0 & 1 & 0 & 0 \\
\hline Hypoplectrus indigo & 1 & 2 & 1 & 0 & 0 & 1 & 0 & 1 \\
\hline Hypoplectrus nigricans & 0 & 0 & 0 & 0 & 0 & 1 & 0 & 0 \\
\hline Hypoplectrus puella & 0 & 3 & 3 & 1 & 7 & 3 & 1 & 1 \\
\hline Lactophrys triqueter & 0 & 0 & 0 & 0 & 0 & 0 & 0 & 1 \\
\hline Lutjanus analis & 0 & 0 & 0 & 0 & 0 & 0 & 0 & 0 \\
\hline Lutjanus apodus & 0 & 6 & 6 & 2 & 4 & 2 & 18 & 2 \\
\hline Lutjanus griseus & 0 & 0 & 0 & 0 & 1 & 0 & 0 & 0 \\
\hline Lutjanus jocu & 0 & 0 & 0 & 0 & 0 & 0 & 0 & 1 \\
\hline Lutjanus mahogoni & 0 & 1 & 2 & 0 & 2 & 1 & 6 & 0 \\
\hline Lutjanus synagris & 0 & 0 & 0 & 2 & 0 & 0 & 0 & 0 \\
\hline Microspathodon chrysurus & 3 & 0 & 0 & 0 & 1 & 0 & 0 & 2 \\
\hline Mulloidichthys martinicus & 0 & 4 & 2 & 1 & 1 & 1 & 21 & 0 \\
\hline Mycteroperca bonaci & 0 & 0 & 0 & 0 & 0 & 0 & 0 & 0 \\
\hline Myripristis jacobus & 1 & 1 & 2 & 0 & 2 & 0 & 0 & 7 \\
\hline Neoniphon marianus & 1 & 5 & 10 & 1 & 7 & 0 & 3 & 1 \\
\hline Ocyurus chrysurus & 0 & 0 & 3 & 3 & 136 & 1 & 9 & 61 \\
\hline Pomacanthus arcuatus & 0 & 1 & 2 & 0 & 1 & 1 & 0 & 0 \\
\hline Pomacanthus paru & 0 & 0 & 2 & 0 & 0 & 0 & 0 & 0 \\
\hline Prognathodes aculeatus & 0 & 1 & 0 & 1 & 2 & 0 & 0 & 0 \\
\hline Pseudopeneus maculatus & 0 & 0 & 0 & 0 & 0 & 1 & 1 & 0 \\
\hline Pterois volitans & 0 & 0 & 0 & 0 & 1 & 1 & 0 & 0 \\
\hline Sargocentron vexillarium & 0 & 0 & 0 & 0 & 0 & 1 & 0 & 0 \\
\hline Scarus iserti & 24 & 27 & 6 & 12 & 41 & 36 & 39 & 10 \\
\hline Scarus taeniopterus & 56 & 47 & 72 & 40 & 82 & 22 & 54 & 83 \\
\hline Scarus vetula & 9 & 1 & 5 & 3 & 2 & 0 & 4 & 8 \\
\hline Serranus tigrinus & 4 & 0 & 0 & 0 & 0 & 0 & 0 & 0 \\
\hline Sparisoma atomarium & 0 & 0 & 12 & 0 & 0 & 0 & 0 & 0 \\
\hline Sparisoma aurofrenatum & 26 & 20 & 22 & 14 & 22 & 24 & 27 & 26 \\
\hline Sparisoma chrysopterum & 0 & 0 & 1 & 0 & 0 & 0 & 0 & 0 \\
\hline Sparisoma rubripinne & 0 & 0 & 0 & 0 & 0 & 1 & 0 & 2 \\
\hline Sparisoma viride & 11 & 12 & 1 & 4 & 7 & 16 & 14 & 8 \\
\hline Stegastes adustus & 0 & 0 & 0 & 0 & 0 & 0 & 0 & 0 \\
\hline Stegastes diencaeus & 16 & 0 & 0 & 1 & 1 & 11 & 4 & 2 \\
\hline Stegastes leucostictus & 1 & 0 & 0 & 1 & 0 & 2 & 0 & 0 \\
\hline Stegastes partitus & 18 & 23 & 30 & 4 & 7 & 4 & 22 & 14 \\
\hline Stegastes planifrons & 3 & 2 & 2 & 6 & 0 & 4 & 0 & 1 \\
\hline Stegastes variabilis & 2 & 0 & 0 & 2 & 1 & 2 & 5 & 0 \\
\hline Synodus synodus & 0 & 0 & 0 & 0 & 1 & 1 & 0 & 0 \\
\hline Thalassoma bifasciatum & 166 & 92 & 0 & 16 & 157 & 24 & 42 & 60 \\
\hline Average abundance & 11.89 & 9.21 & 4.85 & 8.77 & 11.75 & 5.56 & 8.17 & 7.37 \\
\hline Total species number & 34 & 33 & 40 & 33 & 45 & 43 & 35 & 38 \\
\hline
\end{tabular}




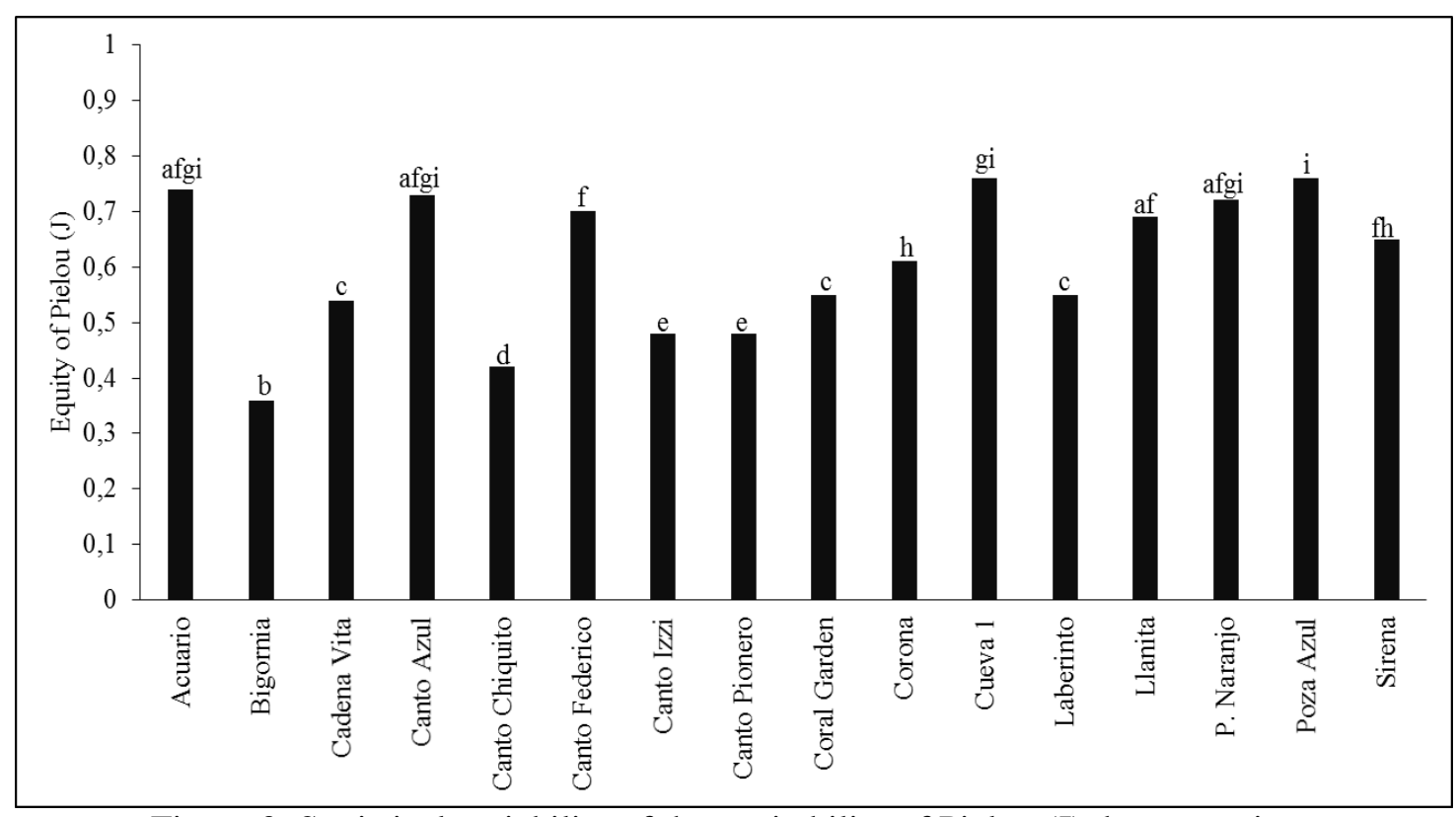

Figure 3: Statistical variability of the equitability of Pielou (J), between sites.

Equal letters represent that there are no statistical differences and different letters that there are differences $(\mathrm{p} \leq 0.0001)$.

Dominance (Fig. 4). Bigornia and Canto Chiquito were the sites with the highest dominance and statistical variability $(\mathrm{p} \leq 0.0001)$ (Tab. 1$)$. The species that most influenced in the dominance at these sites were $C$. cyanea and $C$. parrae respectively. These sites were characterized by a low species richness ( 40 and 47 species, respectively).

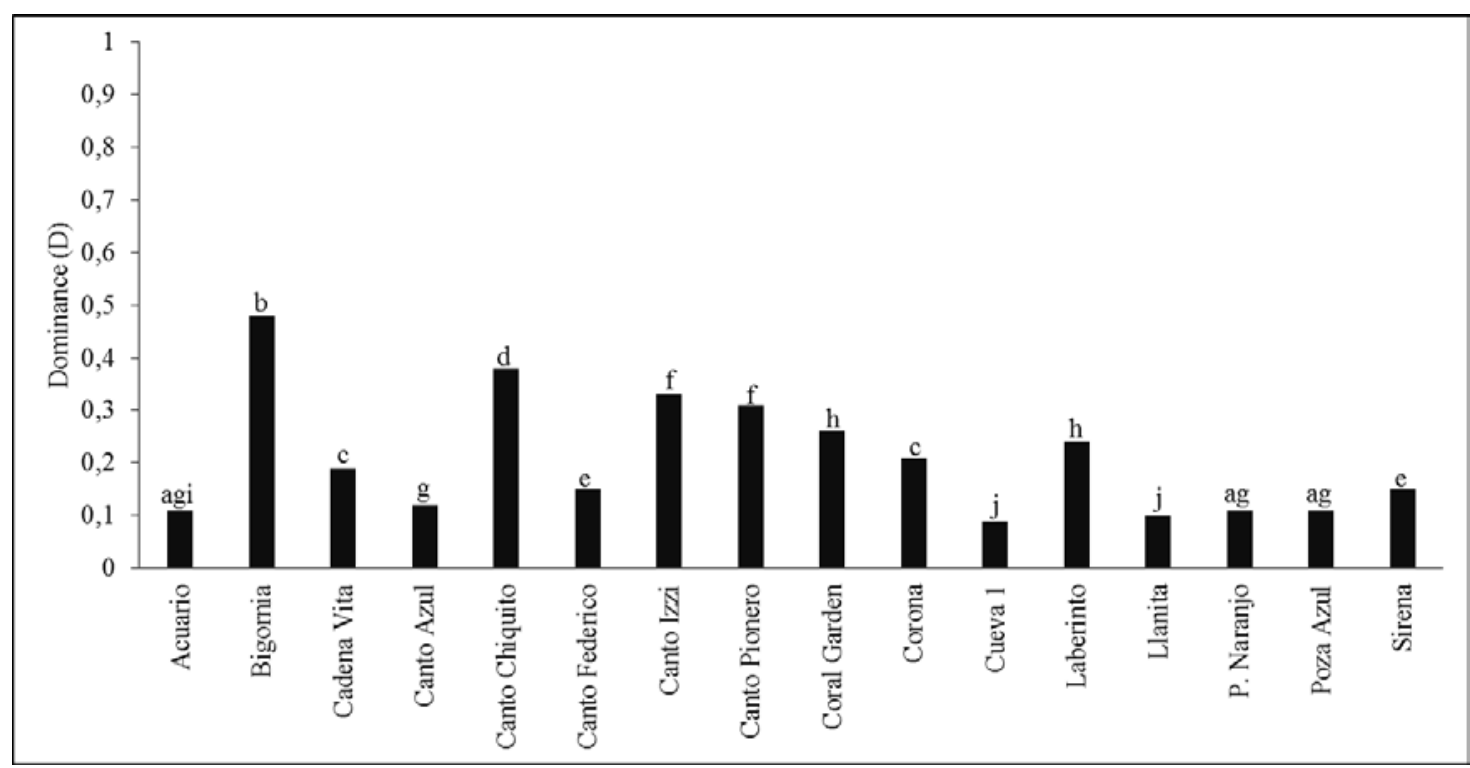

Figure 4: Statistical variability of Dominance (D), between sites. Equal letters represent that there are no statistical differences and different letters that there are differences $(p \leq 0.0001)$. 
Fisher's alpha diversity (Fig. 5). The highest values were recorded in Punta Naranjo (11.49) and Cueva 1 (10.94), which presented the highest number of species with the highest densities (Tabs. 1 and 2). The rest of the sites have few ichthyologic species with significant densities. The species with the highest densities in both sites were C. parrae, C. cyanea, S. taeniopterus, and S. aurofrenatum (Tab. 2). These sites did not show statistical differences between them, but with the other collection sites ( $\mathrm{p} \leq 0.0001)$ (Fig. 5). The lowest diversities were recorded in Bigornia, Coral Garden, Canto Pionero, and Corona (Tab. 1) and in turn the sites with the highest statistical variability for this ecological index $(\mathrm{p} \leq 0.0001)$.

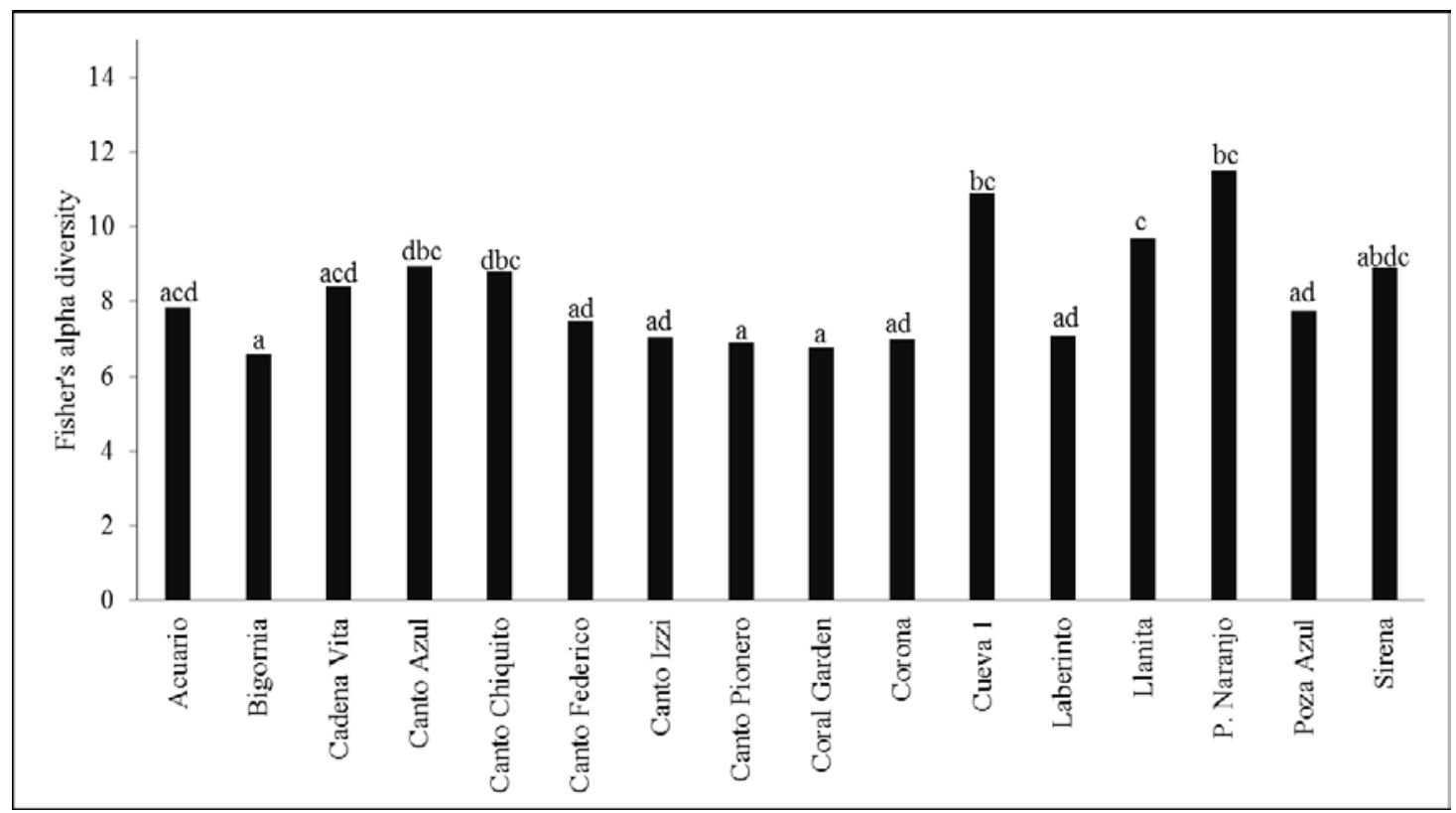

Figure 5: Statistical variability of Fisher alpha diversity, between sites.

Equal letters represent that there are no statistical differences and different letters that there are differences $(\mathrm{p} \leq 0.0001)$.

Beta diversity. Wittaker's global Beta diversity index was (1.26). The sites with the least replacement of species were Acuario with Bigornia (0.41), Cadena de Vita (0.41), Canto Chiquito (0.42), Laberinto (0.40), and Punta Naranjo (0.43).

The species not shared between Acuario with the aforementioned sites were Acanthostracion polygonia, Anisotremus virginicus, Cantherhines macrocerus, Caranx ruber, C. parrae, H. puella, Microspathodon chrysurus, Mulloidichthys martinicus, Myripristis jacobus, Sparisoma rubripinne, Stegastes adustus, and Stegastes variabilis.

Other sites that shared fewer species were Canto Azul with Coral Garden 0.40 highlighting Abudefduf saxatilis, A. quadricornis, and Acanthurus chirurgus. 
Table 3a: Beta diversity in the ichthyologic communities in the contemplative diving sites in Holguín, Cuba.

\begin{tabular}{|c|c|c|c|c|c|c|c|c|}
\hline Sites & 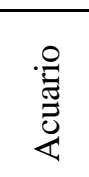 & 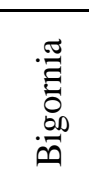 & $\frac{\pi}{\tilde{U}}$ & 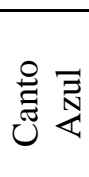 & 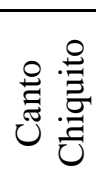 & 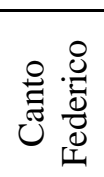 & 惫 & 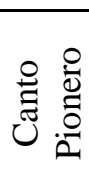 \\
\hline Acuario & 0 & 0.41 & 0.41 & 0.34 & 0.42 & 0.32 & 0.35 & 0.33 \\
\hline Bigornia & 0.41 & 0 & 0.26 & 0.39 & 0.30 & 0.26 & 0.23 & 0.36 \\
\hline Cadena & 0.41 & 0.26 & 0 & 0.27 & 0.20 & 0.30 & 0.25 & 0.22 \\
\hline Canto Azul & 0.34 & 0.39 & 0.27 & 0 & 0.31 & 0.31 & 0.31 & 0.18 \\
\hline Canto Chiquito & 0.42 & 0.30 & 0.20 & 0.31 & 0 & 0.32 & 0.35 & 0.23 \\
\hline Canto Federico & 0.32 & 0.26 & 0.30 & 0.31 & 0.32 & 0 & 0.29 & 0.30 \\
\hline Canto Izzis & 0.35 & 0.23 & 0.25 & 0.31 & 0.35 & 0.29 & 0 & 0.27 \\
\hline Canto Pionero & 0.33 & 0.36 & 0.22 & 0.18 & 0.23 & 0.30 & 0.27 & 0 \\
\hline Coral Garden & 0.29 & 0.32 & 0.33 & 0.40 & 0.37 & 0.29 & 0.38 & 0.30 \\
\hline Corona & 0.37 & 0.31 & 0.30 & 0.24 & 0.36 & 0.37 & 0.34 & 0.32 \\
\hline Cueva 1 & 0.38 & 0.35 & 0.21 & 0.23 & 0.27 & 0.35 & 0.35 & 0.25 \\
\hline Laberinto & 0.40 & 0.25 & 0.35 & 0.33 & 0.39 & 0.31 & 0.37 & 0.32 \\
\hline Llanita & 0.36 & 0.26 & 0.21 & 0.18 & 0.25 & 0.28 & 0.34 & 0.22 \\
\hline Punta Naranjo & 0.43 & 0.32 & 0.24 & 0.34 & 0.30 & 0.40 & 0.35 & 0.23 \\
\hline Pza Azul & 0.36 & 0.27 & 0.21 & 0.29 & 0.28 & 0.27 & 0.33 & 0.28 \\
\hline Sirena & 0.30 & 0.36 & 0.31 & 0.29 & 0.33 & 0.36 & 0.33 & 0.31 \\
\hline
\end{tabular}

Table 3b: Beta diversity in the ichthyologic communities in the contemplative diving sites in Holguín, Cuba.

\begin{tabular}{|c|c|c|c|c|c|c|c|c|}
\hline Sites & 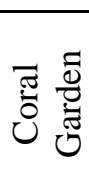 & 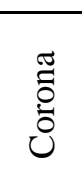 & 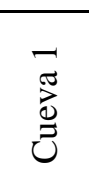 & $\begin{array}{l}\text { 임 } \\
\text { : } \\
\text { 苂 }\end{array}$ & $\begin{array}{l}\stackrel{\widetilde{Z}}{\overparen{\Xi}} \\
\text { 売 }\end{array}$ & 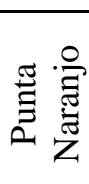 & $\begin{array}{l}\bar{N} \\
\underset{\mathbb{N}}{\mathbb{N}} \\
\text { D }\end{array}$ & $\stackrel{\widetilde{\Xi}}{\mathscr{\varpi}}$ \\
\hline Acuario & 0.29 & 0.37 & 0.38 & 0.40 & 0.36 & 0.43 & 0.36 & 0.30 \\
\hline Bigornia & 0.32 & 0.31 & 0.35 & 0.25 & 0.26 & 0.32 & 0.27 & 0.36 \\
\hline Cadena & 0.33 & 0.30 & 0.21 & 0.35 & 0.21 & 0.24 & 0.21 & 0.31 \\
\hline Canto Azul & 0.40 & 0.24 & 0.23 & 0.33 & 0.18 & 0.34 & 0.29 & 0.29 \\
\hline Canto Chiquito & 0.37 & 0.36 & 0.27 & 0.39 & 0.25 & 0.30 & 0.28 & 0.33 \\
\hline Canto Federico & 0.29 & 0.37 & 0.35 & 0.31 & 0.28 & 0.40 & 0.27 & 0.36 \\
\hline Canto Izzis & 0.38 & 0.34 & 0.35 & 0.37 & 0.34 & 0.35 & 0.33 & 0.33 \\
\hline Canto Pionero & 0.30 & 0.32 & 0.25 & 0.32 & 0.22 & 0.23 & 0.28 & 0.31 \\
\hline Coral Garden & 0 & 0.37 & 0.37 & 0.34 & 0.34 & 0.40 & 0.36 & 0.33 \\
\hline Corona & 0.37 & 0 & 0.23 & 0.30 & 0.25 & 0.31 & 0.29 & 0.29 \\
\hline Cueva 1 & 0.37 & 0.23 & 0 & 0.36 & 0.24 & 0.34 & 0.28 & 0.30 \\
\hline Laberinto & 0.34 & 0.30 & 0.36 & 0 & 0.28 & 0.36 & 0.23 & 0.35 \\
\hline Llanita & 0.34 & 0.25 & 0.24 & 0.28 & 0 & 0.29 & 0.25 & 0.27 \\
\hline Punta Naranjo & 0.40 & 0.31 & 0.34 & 0.36 & 0.29 & 0 & 0.28 & 0.38 \\
\hline Pza Azul & 0.36 & 0.29 & 0.28 & 0.23 & 0.25 & 0.28 & 0 & 0.38 \\
\hline Sirena & 0.33 & 0.29 & 0.30 & 0.35 & 0.27 & 0.38 & 0.28 & 0 \\
\hline
\end{tabular}


Punta Naranjo was the other site that shared fewer species with Canto Federico and Coral Garden 0.40 . The species with the lowest replacement were A. coeruleus, $C$. rostrata, $C$. ruber, C. ocellatus, C. personatus, G. loreto, $H$. bivittatus, $H$. adscensionis, $H$. gummigutta, $H$. nigricans, L. apodus, L. mahogoni, M. martinicus, $P$. arcuatus, and $P$. maculatus. The places that presented a higher degree of species replacement were Canto Azul, with Canto Pionero and La Llanita, sharing 29 species with abundance between one to 813 individuals $100 \mathrm{~m}^{2}$ (Tabs. 2a and 2b).

Gamma diversity. A value of $87.93 \%$ was obtained in this research for the community complex.

Ichthyologic similarity (Fig. 8). The analysis of the similarity between the studied sites regarding the densities of the ichthyologic species allowed to establish four groups. The first made up of Laberinto and less similarity 18\%. The second group with Canto Federico and La Llanita, with $74 \%$ similarity. The third structured with the highest number of sites and similarities above $80 \%$. The fourth group constituted by Canto Azul and Canto Izzi 82\%, Cueva 1 and Acuario with 84\% (Fig. 6). The obtained cophenetic correlation was $92 \%$.

The statistical comparison between the four groups established in this research analysis of similarity allowed obtaining a value of the level of significance $p=0.95$, and a strength of the factors on the samples $\mathrm{R}=0.02$, with no statistical differences between the four groups. Similar results were obtained in independent analysis between each of the groups.

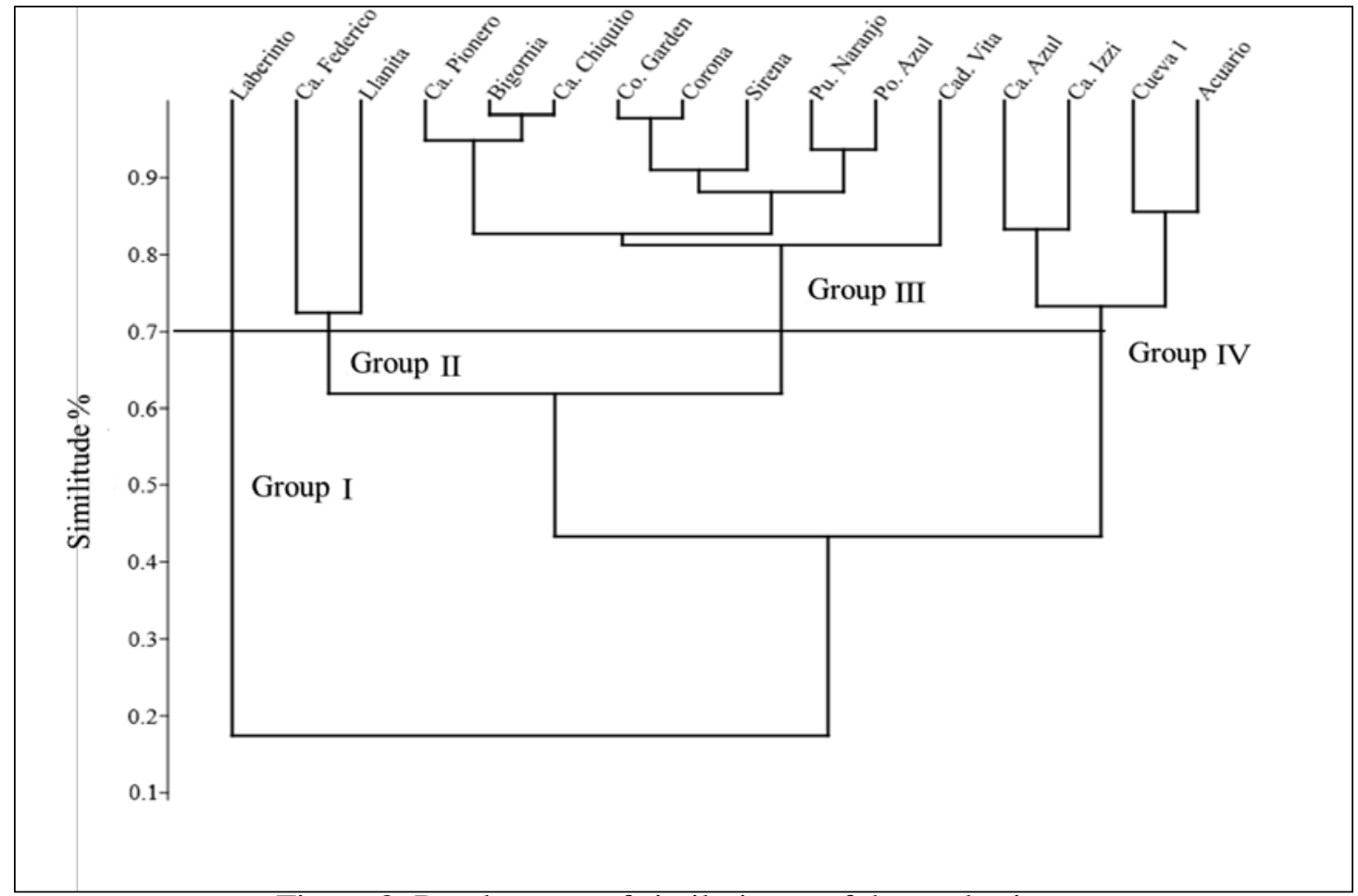

Figure 8: Dendrogram of similarity \% of the study sites

from the density of species for $100 \mathrm{~m}^{2}$,

Cophenetic correlation $=0.92$. 
The species that contributed the most to $80 \%$ dissimilarity were C. parrae $35.11 \%$, C. cyanea $16.46 \%$, T. bifasciatum $7.22 \%$, A. virginicus $4.49 \%, H$. carbonarium $2.90 \%$, S. taeniopterus $2.87 \%$, O. chrysurus $2.76 \%$, $H$. flavolineatum $2.57 \%$, C. personatus $2.53 \%$, H. aurolineatum $2.50 \%$. The roughness of the bottom averaged $21.9 \mathrm{~cm}$, with the highest value in Canto Pionero $(41.9 \mathrm{~cm})$, and the lowest in Canto Federico $(8.5 \mathrm{~cm})$.

The Guardalavaca Holguín coral reef displays a richness of species with an average value of 37.56 species. The average abundances of herbivorous fish (parrots and barbers), and carnivores (snappers, groupers, and grunts) were extremely low (25 and seven individuals in $100 \mathrm{~m}^{2}$, respectively (Tabs. 2a and 2b). The general equity in all the studied sites is poor with values between 0.36 and 0.76 . Dominance presented values between 0.10 and 0.48 . An average Fisher's alpha diversity of 8.24 was obtained. These results allow to infer a poor state of conservation of the fish communities that inhabit this coastal sector.

These environments are characterized by a higher habitat heterogeneity with the dominance of coral species Agaricia agaricites 38.7 ind./10 $\mathrm{m}^{2}$, Millepora alcicornis 27.3 ind. $/ 10 \mathrm{~m}^{2}$, Siderastrea siderea 25.8 ind. $/ 10 \mathrm{~m}^{2}$, and Stephanocoenia intercepta 27.3 ind. $/ 10 \mathrm{~m}^{2}$ (Busutil et al., 2015). The families Scaridae and Haemulidae were those that contributed in a greater number of species in both sampling sites, highlighting the Scarus taeniopterus species as the most abundant. Species richness is an ecological index that allows defining these sites with the best environmental conditions to support a greater number of taxa, among them greater availability of food and number of refuges stand out (Moreno, 2001).

Diversity is strongly linked to the concept of equity; more ecologically equitable fish communities are more diverse. Diversity has a minimum value when abundance is concentrated in a single species, while the others remain with a single individual, while also having a maximum value when all species are equally common (Moreno, 2001).

The Pielou equity index showed significant differences between the protected marine areas of Guanahacabibes and the Arrecifes Xcalak National Park $(F=25.19, p<0.001)$. Presenting the marine area of Mexico higher values $(0.75 \pm 0.05)$ than the area of Cuba $(0.62 \pm$ 0.06) (Cobián-Rojas et al., 2018).

Results similar to those obtained in the Guanahacabibes area 0.62 were obtained in La Llanita and Canto Federico 0.69 respectively, Sirena 0.65 and Corona 0.61. Equity values close to those obtained in the Arrecifes Xcalak 0.75, National Park were determined in Cueva 1 and Poza Azul 0.76, Acuario 0.74, Canto Azul 0.73, and Punta Naranjo 0.73. The rest of the sites not mentioned presented lower values and statistical differences were obtained between the sites with the highest equity values and those with the lowest equity $(\mathrm{p}=0.0001)$.

Ichthyologic similarity. The analysis of similarity between the different sampled sites in relation to the density of the fish, allowed to establish to Laberinto as the site with the least similarity. Bigornia, Canto Chiquito, Coral Garden and Corona as the sites with the greatest ichthyologic similarity. Sites that shared the highest species composition. No statistical differences were detected between the four groups established for $70 \%$ similarity. This result shows that the contribution of the densities between the sites was not significant. It obtained a value of $92 \%$ of the cophenetic correlation, allowing to establish a high correlation between the distances of the simulated matrix and the real one. This high correlation value allows us to infer a good representation of the individual distances between the sites located in the dendrogram of similarity (Gotelli and Ellison, 2004). 


\section{DISCUSSION}

Species richness. The number of species is the most used measure-to-measure biodiversity in different ecosystems, since it reflects different aspects of ecology (Gaston, 1996; Moreno, 2000). The sites that presented the highest number of species were Cadena de Vita with 47 species distributed in 27 genera, 16 families and Canto Chiquito with 46 species distributed in 31 genera, 19 families. Species richness is an ecological index that allows for defining these sites with the best environmental conditions to support a greater number of taxa, among them greater availability of food and number of refuges stand out (Moreno, 2001).

Species richness is associated with local environmental factors and biotic interactions (Llorente and Morrone, 2001). Acuario, Corona and Coral Garden, were sites of less richness. In these latter sites, two commercial fishing bases (Guardalavaca and Puerto de Vita fishing base) carry out the fishing activity, both made up of approximately 60 vessels (Puga et al., 2018). Where different fishing gear is used, highlighting the nets of $100 \mathrm{~m}$ long and palanqrues of 100 hooks (Baisre, 2018). During the samplings, the occasional presence of people exercising the activity with pneumatic rifles in the work area was verified. This type of fishery is exerted selectively on the large predators and herbivores of the reef (Aguilar, 2005; Aguilar and González, 2007). Underwater fishermen report an average annual catch of approximately $995 \mathrm{~kg}$ in this coastal sector. Baisre (2018) found that $74 \%$ of the fishing sites in Cuba are overexploited and 5\% have collapsed. The overfishing has been strongly correlated with ecological collapse in marine ecosystems, such as the absence of large predators, a characteristic present in the 16 monitored sites (Harborne et al., 2017).

Froese et al. (1998) suggested that the fishing exploitation of multispecies communities has an effect of change in the relative abundance in the different functional fish groups. He stated that the greatest expected effect is the succession of large groupers by smallsized and short-lived herbivorous species. In Cuba, there are more than 60 years of overexploitation of fishing, establishing a significant decrease from the 90s in all fishing sites, with a reduction of $40 \%$ in 21 of the most commercially important species (Baisre, 2018). However, there are dissimilar factors that could be influencing the current condition of the sites with less richness, such as recruitment, climate change and the characteristics of the habitat, and food availability, among others (González and Aguilar, 2000). Another aspect that is considered is the selective fishing carried out in these sites on the ichthyologic communities.

Blaber (1985) found a relationship between the increase in fish richness and the variety of the habitat and the latitudinal changes, in Southeast Africa. Greater species richness may be associated with the high complexity of the relief, which provides abundant refuges and food for fish in Cuba (Cobián et al., 2018), as it has been confirmed by other authors in other regions (Núñez-Lara et al., 2005). Although, in this study the roughness of the bottom of the highest value was obtained in Canto Pionero site with $41.9 \mathrm{~cm}$ and a richness of 41 species, followed by Punta Naranjo $33 \mathrm{~cm}$ and a richness of 50 species and Acuario with $31 \mathrm{~cm}$ and 35 species. Indicating that greater roughness does not necessarily imply favourable ecological conditions in a coral reef. If it is considered that dead corals also increase the relief of the bottom (McField and Richards, 2007). A marked deterioration in the health status of Caribbean coral reefs has been observed, caused by anthropogenic stressors and climate change (Mumby et al., 2014; Graham et al., 2015)

The fish communities present in two marine protected areas, one in Guanahacabibes (Cuba) and the other in the Arrecifes Xcalak National Park (Mexico), these did not present statistical differences for the number of fish species. Presenting the Guanahacabibes area with a higher richness value (43 species) than the Arrecifes Xcalak National Park, (35 species) (Cobián-Rojas et al., 2018). Martínez-Iglesias and Serpa (1997), point out the significantly 
reduced abundance of fish present in Punta Francés, Isla de la Juventud, Cuba. The abundance of herbivorous fish in Cayo Levisa is also extremely low. These authors recorded 77 species of fish, the most abundant being S. taeniopterus, C. cyanea, T. bifasciatum, S. partitus and $G$. loreto (De la Guardia et al., 2005). Similar values obtained by Cobián-Rojas et al. (2018), were obtained in the different study stations of this work. Anthropogenic disturbance in the marine environment can influence the richness of ichthyologic species, being considered as the stress that excludes species at high trophic levels and does not prevent competitive exclusion by superior competitors (Paine, 1966; Huston, 1979; Connell, 1983).

Ichthyologic equity. Equity refers to the equitability in the abundances of the different species; and it is typically evaluated taking into account the number of dominant species in relation to the number of rare or not common species (Magurran, 2004; Moreno et al., 2011). The more species there are in a site, as well as the more homogeneous their distribution, the greater its diversity. The highest equitabilities were obtained in Cueva 1, Poza Azul, Acuario, Canto Azul and Punta Naranjo. Communities with greater equity indicate that they present are stable between the actions of the local biota and abiotic elements, competition, predation and immigration to other locations (Sonco, 2013). The species that most contributed to this ecological index in the aforementioned sites were: A. bahianus, A. coeruleus, C. fulva, $C$. cyanea, H. garnoti, H. tricolor, S. taeniopterus, S. aurofrenatum, and S. partitus. The abundances of these species were extremely low. This result may be indicating that there is a balanced pressure from legal and poaching at sites with close values of the equity index. This result is indicating that there is a balanced pressure of legal and poaching in sites with close values of the equity index (Cobián-Rojas et al., 2018).

Ichthyologic dominance. This index is inverse to the concept of community equity, since it takes into account the most important species without considering the rest of the species, being less sensitive to the richness of the species (Krebs, 1996; Magurran, 2004). Dominance is strongly influenced by the importance of the most dominant or common species (Magurran, 1988; Moreno, 2001), therefore they are more sensitive to changes in equitability (Feinsinger, 2003). The species that most influenced the sites of greatest dominance were $C$. cyanea and C. parrae. This last species was also the most abundant in the Guanahacabibes National Park (Cobián-Rojas et al., 2018). Bigornia and Canto Chiquito were the sites with the highest dominance, due to the fact that they are located on the reef slope (wall edge). This habitat favors a high dominance of a few species (C. parrae, C. cyanea, and S. partitus), which makes the equity lower and, therefore, the diversity index as well (Cobián-Rojas et al., 2018). The same behavior was observed in the reef slope of the Bahía de Cochinos (Cuba) (Chevalier and Cárdenas, 2006).

These two species present high densities in different dive sites in Cuba, such as in the coral reefs of María la Gorda, Guanahacabibes National Park (Cobián and Chevalier, 2009), registering densities for both species of 84.79 and 109.3 ind./100 $\mathrm{m}^{2}$. Values lower than those obtained in this study for Bigornia, Cadena de Vita, Canto Chiquito, Canto Pionero, Coral Garden, Corona, Llanita, and Poza Azul (Tab. 2).

Fisher's alpha diversity. Punta Naranjo and Cueva 1 were the sites with the highest Fisher alpha diversity. They are the sites with the highest number of species and the highest densities. Presenting statistical differences with the places of less diversity. Highlighting the species $C$. parrae, C. cyanea, S. taeniopterus, and $S$. aurofrenatum with the highest abundances. Species diversity is an important indicator in the study of community ecology and conservation biology. A direct relationship has been established between alpha diversity and the functioning of ecosystems, such as productivity and stability (Maclaurin and Sterelny, 
2008). Analysing this ecological index, Punta Naranjo and Cueva 1 constitute the most conserved sites in terms of ichthyologic communities. Bigornia, Coral Garden, Canto Pionero, and Corona places with less diversity. Indicating that the ichthyologic communities that inhabit these places have a poor state of conservation. The diversity is a characteristic that can be measured in marine ecosystems, such as productivity, density, and biomass (Alcolado, 1998). The diversity index reflects the complexity and structure of the system under study (Rodríguez-Zaragoza et al., 2011). The sites with low diversity are the product of fishing in this area, being more intense in the months of September to December, these sites are more accessible to fishermen (Baisre, 2018). Areas in Cuba exposed to intensive fishing and pollution, such as those along the Northwest coast, exhibit reduced coral density and ichthyology diversity (Duran et al., 2018).

Marine ecosystems with greater diversity will have better functioning and resilience (Hughes et al., 2003). The main causes of the loss of diversity are habitat destruction, overexploitation, pollution, climate change, and invasive species (Gutiérrez, 2006; Santos and Tellería, 2006; Capote et al., 2012). In the case of invasive species, the ichthyologic communities that inhabit these sites have been strongly affected by the presence of the lionfish (Pterois volitans), a generalist predator (Reynaldo et al., 2019). Other possible causes of the low fish diversity in the studied sites and the lower densities in the fish communities in the tourism sector of Holguín could be strongly influenced by the tourism and navigation activities themselves (Esslemont, 1999; Rouphael and Inglis, 2000; Zakai and Chadwick-Furman, 2002). As well as legal and illegal extractive and fishing activities (Padilla, 2000; Pikitch et al., 2004). These factors generate a pattern of chronic and constant stress that does not allow the recovery of fish communities (Buddemeier et al., 2004).

Close relationships have been determined between Fisher's alpha diversity and latitude, climate, biological productivity, habitat heterogeneity, habitat complexity, disturbance, and geographic distances (Fischer, 1960, 1961; Pianka, 1966; Stehli et al., 1969; Brown and Gibson, 1983; Stevens, 1989; Ricklefs, 1990). The theory of environmental heterogeneity supports the idea that heterogeneous environments allow them to be colonized by a greater number of species, thereby increasing diversity (Badii and Landeros, 2007). The fishing constitutes the fundamental factor of the reduced ecological diversity of the ichthyological communities in the Guardalavaca reef, Holguín. Overfishing is one of the interventions of man that most alter the diversity of fish in Caribbean coral reefs, by removing herbivorous and carnivorous fish (Bellwood et al., 2004).

In Punta Frances marine park in Cuba, a reduction in the diversity in the ichthyologic communities was obtained as a result of illegal and commercial fishing. Reducing mainly the predatory families, Lutjanidae and Serranidae, as well as the species of the genus Mycteroperca (Angulo et al., 2007). Individuals of S. coelestinus, and S. guacamaia, important grazers of macroalgae, were present in Northwest Cuba up until the 1970 (Aguilar and Gonzalez-Sanson, 2007), species not recorded in this study. But they have been targeted by fishers in recent decades. Their current scarcity has put the region at risk of remaining in a coral-depleted alternative stable state (Steneck et al., 2014).

Abundant fishing areas in the Caribbean Sea are overexploited, including Cuba. These practices have changed the structure of fish communities of Caribbean coral reefs, reducing the abundance of predatory fishes (Valdivia et al., 2017). It is estimates that about 74\% of Cuban fisheries are overexploited, 20\% are fully exploited, and 5\% have collapsed (Baisre, 2018). Groupers and snappers, which aggregate on a few, predictable spawning sites, are especially prone to uncontrolled fishing (Claro et al., 2009). Commercial catches of the Nassau grouper, E. striatus, have declined by $98 \%$ (Jones et al., 2004; Baisre, 2018). 
Beta ( $\beta$ ) diversity. Beta diversity is a measure of the change of species between different types of communities or habitats; as such, it corresponds to the spatial contiguity of different communities or habitats (Magurran, 2004). Halffter et al. (2005), defines Beta diversity as the degree of change or replacement in the composition of species between different communities of a landscape. In the present study, the communities that presented a greater replacement of species had a greater geographic distribution; Canto Azul is located from Canto Pionero at a distance of $16 \mathrm{~km}$ and from La Llanita at $18 \mathrm{~km}$. This result allows inducing that despite being geographically, remote communities show high ecological similarities. Acuario was the site that had the least replacement of species with the Bigornia, Cadena de Vita, Canto Chiquito, Laberinto and Punta Naranjo sites. Like Canto Azul with Coral Garden. In addition to Punta Naranjo with Canto Federico and Coral Garden.

There are species that share the same natural resource between different sites and others that interact negatively with other species to obtain this limited resource. This resource can be food or shelter, modifying the structure, dynamics and productivity of the communities (Badii and Landeros, 2007). Fish communities that share a greater number of species are susceptible to interspecific competition. Competition between species is the driving force in the structure of communities, involving as a factor increasing levels of diversity in a given area (Badii and Landeros, 2007). The increase in temperature has an obvious direct effect on the displacement of the geographical limits of many ichthyological species (Southward and Boalch, 1994; Southward et al., 1995; Alcock, 2003). During this research sampling activities, elevated temperatures between 30 and $32^{\circ} \mathrm{C}$ were obtained. There is an increase in these temperatures in the last five years (INSMET, 2020). This is a possible cause of the replacement of the species between the different sites studied.

Gamma diversity. The gamma diversity is the number of species in the set of sites or communities that make up a landscape (Forman and Gordon, 1986). Whittaker (1972) defined it as the species richness of a set of communities that make up a landscape, resulting from both alpha and beta diversities. The result obtained in this research allows us to establish that alpha diversity comprises $42.71 \%$ and beta diversity $57.29 \%$ of the gamma diversity of the studied marine ichthyologic communities. All communities are structured mainly at the ecological level by the degree of differentiation between them or the degree of replacement of species through environmental gradients (Moreno, 2001). In this way, we can define that those communities with a greater replacement of species are the ones that should be more conserved, being Canto Azul, Canto Pionero, and La Llanita.

The fish species that most influenced the dissimilarity between Laberinto and the different sites were: C. parrae, C. cyanea, T. bifasciatum, A. virginicus, $H$. carbonarium, S. taeniopterus, O. chrysurus, H. flavolineatum, C. personatus, and $H$. aurolineatum. The species that contributed the most to group III similarity with the highest number of sites were: A. bahianus, A. coeruleus, C. fulva, C. capistratus, C. cyanea, C. parrae, $H$. flavolineatum, $H$. garnoti, $H$. tricolor, $S$. iserti, S. taeniopterus, S. aurofrenatum, $S$. viride, and $T$. bifasciatum. These species are specialized herbivores that constitute key elements in the structure and composition of fish communities in coral reefs; controllers of the overgrowth of algae on the coral reef (Williams and Polunin, 2001). The group IV made up of four sites had a greater contribution of the similarity of the species mentioned above in addition to C. cruentata, H. plumieri, H. rufus, L. apodus, and S. partitus. 
Key fish in the control and growth of vegetation cover on the seabed (Williams and Polunin, 2001). The structure of ichthyologic communities includes ecological interactions that produce patterns (spatial, temporal, cyclical, and ordered) of distribution and abundance (Kikkawa, 1986). The number of interconnections in a community depends mainly on the interacting species. Among these interconnections, the interspecific competition, the trophic, and symbiotic interrelationship nested in trophic networks stand out. Increasing the complexity increases the stability of the community. The stability of fish communities depends on four principal components, which are the magnitude, frequency, elasticity (resilience), and the persistence of the species (Badii and Landeros, 2007).

In the dive sites of Holguín, Cuba, the greatest richness of ichthyologic species was obtained in Canto Izzi. The fish community with the highest equitableness and alpha Fisher diversity was recorded in Cueva 1, presenting a greater Bigornia ecological dominance. The sites with the highest species replacement were Canto Azul with Canto Pionero and La Llanita. The set of communities in these sites is structured mainly by the replacement of species. No statistical differences were found with respect to the density of the species between the different sampling stations.

\section{CONCLUSIONS}

In the Holguín reef, the ichthyologic communities have low species richness, equitableness and alpha Fisher diversity. We speculate that this difference could be related to commercial and illegal overexploitation of fishing in the coral reef, however, there is no direct, empirical evidence to support this assertion.

The greatest richness of ichthyologic species was obtained in Canto Izzi (47) area. The fish community with the highest equitableness and alpha Fisher diversity was recorded in Cueva 1 (11) area, presenting a greater Bigornia ecological dominance (0.48).

The results suggest the poor state of conservation of the fish communities present in the Guardalavaca reef, Holguín, Cuba. 


\section{ACKNOWLEDGEMENTS}

To all the staff of the "Eagle Ray" Diving Center, Guardalavaca beach Cuba, for providing us with the autonomous diving equipment, the boat, and its facilities. 


\section{REFERENCES}

1. Alcock R., 2003 - The effects of climate change on rocky shore communities in the bay of Biscay, 1895-2050, Southampton, 21.

2. Aguilar C. and González S. G., 2007 - Composición de la ictiofauna costera de Ciudad de la Habana y evaluación preliminar de los factores que la determinan, Revista de Investigaciones Marinas, 28, 1, 43-56. (in Spanish)

3. Aguilar C., 2005 - La ictiofauna costera de Ciudad de La Habana: Efectos acumulativos de agentes estresantes múltiples en varios niveles de organización biológica, Thesis presented as an option to the scientific degree of Doctor in Biological Sciences, Centro de Investigaciones Marinas, Universidad de La Habana, Cuba, 122. (in Spanish)

4. AGRRA, 2000 - The AGRRA, Rapid Assessment Protocol, Atlantic and Gulf rapid reef assessment methodology, 9, URL: http://coral.aoml.noaa.gov/agrra/methodhome.htm.

5. Angulo V. J., Borrego R. A., Borrego H. R. and Gonzáles S. G., 2007 - Effects of tourism activities on coral reef communities in the Punta Frances national marine park, Cuba, Revista Investigaciones Marinas, 28, 2, 159-175.

6. $\quad$ Alcolado P. M., 1998 - Conceptos e índices relacionados con la diversidad, Avicennia, 8, 9, 721. (in Spanish)

7. Alcolado P. M. and Durán A., 2011 - Sistema de escalas para la clasificación y puntaje de condición del bentos e ictiofauna de arrecifes coralinos de Cuba y del Gran Caribe, Serie Oceanológica, 8, 25-29. (in Spanish)

8. Aviles-Torres S., Schmitter-Soto J. J. and Barrientos-Medina R. C., 2001 - Patrones espaciales de la riqueza de peces en lagunas costeras del sur de Quintana Roo, México, Hidrobiológica, 11, 2, 141-148. (in Spanish)

9. Ayala-Pérez L. A., Terán-Gonzáles G. J., Flores-Hernández D., Rams-Miranda J. and SosaLópez A., 2012 - Spatial and temporal variability of fish community abundance and diversity off the coast of Campeche, Mexico, Latin American Journal of Aquatic Research, 40, 1, 63-78.

10. Badii M. H. and Landeros J., 2007 - Invasión de especies o el tercer jinete de Apocalipsis ambiental, Una amenaza a sustentabilidad Daena, 2, 1, 39-53, www.daenajournal.org. (in Spanish)

11. Bellwood D. R. and Hughes T. P., 2001 - Regional-scale assembly rules and biodiversity of coral reefs, Science, 292, 1532-1534.

12. Begon M., Townsend C. R. and Harper J. L., 2006 - The influence of population interactions on community structure, 550-577, in Ecology from individuals to ecosystems, Cuarta Edición, Blackwell, Publishing Ltd., 738.

13. Baisre J. A., 2018 - An overview of Cuban commercial marine fisheries: the last 80 years, Bulletin Marine Sciences, 94, 2, 359-375, https://doi.org/10.5343/bms.2017.1015.

14. Blaber S. J. M., 1985 - The ecology of fishes of estuaries and lagoons of the Indopacifíc with particular referente to Southeast Africa, Chapter 12, 247-266, in Fish community ecology in estuaries and coastal lagoons, towards an ecosystem integration, Yaiiez-Arancibia A. (ed.), UNAM, Mexico, 654.

15. Bellwood D. R., Hughes T. P. and Nyström M., 2004- Confronting the coral reef crisis, Nature, 429, 6994, 827-833.

16. Brock V. E., 1954 - A preliminary report on a method of estimating reef fish's populations, Journal Wildlife Management, 18, 3, 297-308.

17. Brower J., Zar J. and von Ende C. N., 1997 - Field and laboratory methods for general ecology, McGraw-Hill, Boston, ISBN-13: 978-0697243584, 288.

18. Brown J. H. and Gibson A. C., 1983 - Biogeography, St. Louis, Mosby Company, ISBN 08016-0824-4.

19. Buddemeier R. W., Kleypas J. A. and Aronson R. B., 2004 - Coral reefs and global climate change: potential contributions of climate change to stresses on coral reef ecosystems, Pew Centre for Global Climate Change, www.pewclimate.org, 44. 
20. Busutil L., Reynaldo-de la Cruz. E., García-Férnandez F., González-Sánchez P. M., RodríguezMachado S. and Vega-Torres. A., 2015 - Evaluación del estado de conservación de los sitios de buceo comtemplativo en el sector costero turístico de Holguín, Cuba, Servicio científico Técnico, CISAT-CITMA, 86. (in Spanish)

21. Caballero H., Chevalier P. P., Varona G., Cárdenas A. L., Pastor L., Pérez-Hernández A. and García Y., 2006 - Componentes más comunes de la fauna del arrecife de coral de la costa oriental de Bahía de Cochinos, Cuba, corales, esponjas, gorgonaceos y peces, Revista de Investigaciones Marinas, 25, 1, 37-44. (in Spanish)

22. Campos C. A., 2012 - Efecto de la invasión del Pez León (Pterois volitans) sobre los patrones de coexistencias en las comunidades de peces marinos, Diploma Thesis, Universidad de Oriente, Santiago de Cuba, 77. (in Spanish)

23. Capote R. P., Mitrani-Arenal I. and Suárez A. G., 2012 - Conservación de la biodiversidad cubana y cambio climático en el archipiélago cubano, Anales de la Academia de Ciencias de Cuba, 1, 1-25. (in Spanish)

24. Chevalier P. and Cárdenas A. C., 2006 - Estudio diagnóstico del arrecife coralino del Rincón de Guanabo, Ciudad de La Habana, Cuba, 2, Peces, Revista de Investigaciones Marinas, 27, 2, 121-132. (in Spanish)

25. Clarke K. R., 1993 - Non-parametric multivariate analysis of changes in community structure, Australian Journal of Ecology, 18, 117-143.

26. Claro R., 2006 - La biodiversidad marina de Cuba, Instituto de Oceanología, 256.

27. Claro R. and García-Arteaga J. P., 1994 - Estructura de las comunidades de peces en los arrecifes del grupo insular Sabana-Camagüey, Cuba, Avicennia, 2, 83-107. (in Spanish)

28. Claro R., Sadovy-de Micheson Y., Lindeman K. C. and García-Cagide A. R., 2009 - Historical analysis of Cuban commercial fishing effort and the effects of management interventions on important reef fishes from 1960-2005, Fish Research, 99, 7-16, https://doi.org/10.1016/j.fihres.2009.04.004.

29. Claro R. and García-Arteaga P., 2001 - La ictiofauna de los fondos blandos del archipiélago, Sabana-Camagüey, Cuba, Revista de Investigaciones Marinas, 22, 2, 117-127. (in Spanish)

30. Cobián R. D. and Chevalier M. P., 2009 - Evaluación de las asociaciones de peces de los arrecifes coralinos del centro internacional de buceo maría la gorda, parque nacional Guanahacabibes, Cuba, Revista Ciencias Marinas y Costeras, 1, 6, 111-125, DOI: 10.15359/revmar.1.6. (in Spanish)

31. Connell J. H., 1983 - On the prevalence and relative importance of interspecific competition: evidence from field experiments, American Naturalist, 122, 661-696.

32. Del Monte-Luna P. and Lluch-Belda D., 2016 - Approaching the potential of world marine fisheries, Transylvanian Review of Systematical and Ecological Research, 18.1, The Wetlands Diversity, 45-56, DOI: 10.1515/trser-2015-0079.

33. De la Guardia González-Díaz P., Valdivia A. and González-Sansón G., 2005 - Características generales de los arrecifes coralinos en la zona de buceo de Cayo Levisa, Archipiélago de los Colorados, Cuba, Revista de Investigaciones Marinas, 26, 1, 37-44. (in Spanish)

34. Duran A., Shantz A. A., Burkepile D. E., Collado-Vides L., Ferrer V. M., Palma L., Ramos A. and González-Díaz P., 2018 - Fishing, pollution, climate change, and the longterm decline of coral reefs of Havana, Cuba, Bulletin Marine Sciences, 94, 2, 213-228, https://doi.org/10.5343/bms.2017.1061

35. Esslemont G., 1999 - Heavy metals in corals from Heron Island and Darwin Harbour, Australia, Marine Pollution Bulletin, 38, 1051-1054.

36. Feinsinger P., (ed.) 2003 - El diseño de estudios de campo para la conservación de la biodiversidad, FAN, Santa Cruz Bolivia, 242. (in Spanish)

37. Fischer A. G., 1960 - Latitudinal variation in organic diversity, Evolution, 14, 64-81.

38. Fischer A. G., 1961 - Latitudinal variation in organic diversity, America Sciences, 49, 50-74. 
39. Forman R. T. and Gordon M. (eds), 1986 - Landscape ecology, John Wiley and Sons, New York, 1-169.

40. Froese R., 1998 - Length-weight relationships for 18 less-studied fish species, Journal Application Ichthyology, 14, 117-118.

41. Gaston K. J. (ed.), 1996 - Species richness: measure and measurement, in Biodiversity, a biology of numbers and difference, Blackwell Science, Cambridge, 77-113.

42. González S. G. and Aguilar C., 2000 - Influencia de la contaminación de la Bahía de La Habana (Cuba) sobre las asociaciones de peces costeros, 2, Análisis multidimensional, Revista de Investigaciones Marinas, 21, 1-3, 60-70. (in Spanish)

43. González-Sansón G. and Betancourt C. A., 2003 - Variaciones espaciales y temporales en la abundancia de las especies dominantes de peces de arrecife de coral en la costa de ciudad de La Habana, Cuba, Revista de Investigaciones Marinas, 24, 2, 99-110. (in Spanish)

44. Gotelli N. J. and Ellison A. M., 2004 - A primer of ecological statistics, Sunderland: Sinauer Associates, 102.

45. Graham N. A. J., Jennings S., MacNeil A. A., Mouillot D. and Wilson S. K., 2015 - Predicting climate-driven regime shifts versus rebound potential in coral reefs, Research Letter Naturalist, 518, 94-97, https://doi.org/10.1038/nature14140.

46. Gutiérrez F., 2006 - Estado de conocimiento de especies invasoras, Propuesta de lineamientos para el control de los impactos, Bogotá: Instituto de Investigación de Recursos Biológicos Alexander von Humboldt, 576. (in Spanish)

47. INSMET, 2020 - Pronóstico mensual de totales de precipitación y temperaturas medias extremas del archipiélago de cuba para septiembre de 2020, Copyright (C) 1997-2020, INSMET (B) Todos los derechos reservados, http://www.insmet.cu. (in Spanish)

48. Jones L., Warner G., Linton D., Alcolado P., Claro-Madrua R., Clerveaux W., Estrada R., Fisher T., Lockhart K. and Pardee M., 2004 - Status of coral reefs in the Northern Caribbean and Western Atlantic node of the GCRMN, in Status of coral reefs of the world, Wilkinson C. R. (ed.), Townsville, Austrailia, Global Coral Reef Monitoring Network and Australian Institute of Marine Science, 451-472.

49. Halffter G., Soberon J., Koleff P. and Melic A., 2005 - Sobre diversidad biológica: el significado de las diversidades alfa, beta y gamma, Guadalajara-México, m3m-Monografía 3er Milenio, 4, 1-242. (in Spanish)

50. Hammer Ø., Harper D. A. T. and Ryan P. D., 2001 - PAST: Paleontological Statistics Software Package for Education and Data Analysis, Palaeontologia Electronica, 4, 1-9.

51. Harborne R. A., Rogers A., Yves-Marie B., Mumby P. J., 2017 - Multiple stressors and the functioning of coral reefs, Annual Review of Marine Science, 9, 1, 445-468.

52. Horn H. S., 1966 - Measurement of overlap in comparative ecological studies, American Naturalist, 100, 419-424.

53. Hughes T. P., Baird A. H., Bellwood D. R., Card M., Connolly S. R., Folke, C. and Lough J. M., 2003 - Climate change, human impacts, and the resilience of coral reefs, Science, 301, 5635, 929-933, DOI: 10.1126/science.1085046.

54. Humann P. and Deloach N., 2002 - Reef fish identification (Florida-Caribbean-Bahamas), 3rd edition, New World Publications, 259.

55. Huston M., 1979 - A general hypothesis of species diversity, American Naturalist, 113, 1, 81101.

56. Kikkawa J., 1986 - Complexity, diversity and stability, 41-62, in Community Ecology Pattern and Process, Kikkawa J. and Anderson D. J. (eds), Blackwell Scientific Publications, Mass.

57. Krebs C. J., 1996 - Ecological methodology, Harper Collines Publishers, New York, N.Y, 654.

58. La O. J. A., Salinas E. and Licea J. E., 2012 - Aplicación del diagnóstico geoecológico del paisaje en la gestión del turismo litoral: Caso Destino Turístico Litoral Norte de Holguín, Cuba, Investigaciones Turísticas, 3, 1-18. (in Spanish)

59. Landen R., 1996 - Statistics and partitioning of species diversity, and similarity among multiple communities, Oikos, 76, 1, 5-13. 
60. Llorente B. J. and Morrone J. J., 2001 - Introducción a la biogeografía en Latinoamérica: teorías, concepto, métodos y aplicaciones, Facultad de Ciencia, U.N.A.M. México D.F, 137. (in Spanish)

61. Maclaurin J. and Sterelny K., 2008 - What is biodiversity? The University of Chicago Press, Chicago, 224.

62. Magurran A. E., 1988 - Ecological diversity and its measurement, Princeton University Press, New Jersey, 179.

63. Magurran A. E., 2004 - Measuring biological diversity, Nueva York: John Wiley and Sons 306, McField M. and Richards K. P., 2007 - Healthy reefs for healthy people: a guide to indicators of reef health and social well-being in the Mesoamerican reef region, with contributions from Gorrez M. and McPherson M., 208.

64. Martínez-Iglesias J. C. and Serpa A., 1997 - Informe del diagnóstico ecológico de los principales ecosistemas marinos en Punta Francés, Isla de la Juventud, informe técnico, Instituto de Oceanología, 16.

65. McField M. and Richards K. P., 2007 - Healthy reefs for healthy people: a guide to indicators of reef health and social well-being in the Mesoamerican reef region, with contributions from Gorrez M. and McPherson M., 208.

66. Moreno C. E., 2000 - Diversidad de quirópteros en un paisaje del centro de Veracruz, México, Thesis presented as an option to the scientific degree of Doctor in Biological Sciences, Instituto de Ecología, Xalapa A. C., Ver., México, 150.

67. Moreno C. E., 2001 - Métodos para medir la biodiversidad, M. and T.-Manuales y Tesis SEA, 1, Zaragoza, 84.

68. Moreno E. C., Barragán F., Pineda E. and Pavón P. N., 2011 - Reanálisis de la diversidad alfa: alternativas para interpretar y comparar información sobre comunidades ecológicas, Revista Mexicana de Biodiversidad, 82, 1249-1261. (in Spanish)

69. Morisita M., 1959 - Measuring interspecific association and similarity between communities, University Series Edition Biology, 3, 65-80.

70. Mumby P. J., Flower J., Chollett I., Box S. J., Bozec Y. M., Fitzsimmons C., Forster J., Gill D., Griffith-Mumby J. and Oxenford H. A., 2014 - Towards reefs resilience and sustainable livelihoods: a handbook for Caribbean coral reefs managers, University of Exeter, Exeter, 172.

71. Núñez-Lara E., Arias-onzález J. E. and Legendre P., 2005 - Spatial patterns of Yucatan reef fish communities: Testing models using a multi-scale survey design, Journal of Exploratory Marine Biology and Ecolology, 324, 157-169.

72. Padilla C., 2000 - Evaluación del potencial de uso sustentable del coral negro en Isla Cozumel y parte sur de Quintana Roo, México: reporte técnico, Puerto Morelos, inp/fmcn, 70. (in Spanish)

73. Paine R. T., 1966 - Food web complexity and species diversity, America Naturalist, 100, 65-75.

74. Pianka E. R., 1966 - Latitudinal gradients in species diversity: a review of concepts, America Naturalist, 100, 33-46.

75. Pikitch E., Santora C., Babcock E., Bakun A., Bonfil R., Conover D., Dayton P., Doukakis P., Fluharty D. and Heneman B., 2004 - Ecosystem-based fishery management, Science, 305, 346347.

76. Puga R., Valle S., Kritzer J. P., Delgado G., de León M. E., Giménez E., Ramos I., Moreno O. and Karr K. A., 2018 - Vulnerability of nearshore tropical fifih in Cuba: implications for scientifi and management planning, Bulletin Marine Sciences, 94, 2, 377-392, https://doi.org/10.5343/bms.2016.1127.

77. Reynaldo E., Vega A., Fernández A., Cruz J., Córdova E. and Cruz P., 2018 - Distribución y abundancia del pez león Pterois volitans en la zona costera de uso turístico de Holguín, Cuba, Novitates Caribaea, 12, 63-73. (in Spanish)

78. Reynaldo E., Vega T. A., Vega C. M. E., Fernández V. A., Cruz R .J., Córdova G. E. and Cruz R. P., 2019 - Preferencias tróficas de Pterois volitans (Scorpaenidae) en el área costera de Holguín, Cuba, Novitates Caribaea, 13, 1-12. (in Spanish) 
79. Ricklefs R. E., 1990 - Ecology, 3rd edition, New York: W. H. Freeman, 178.

80. Rodríguez R. J., Abitia C. L. A., Galván M. F. and Chávez R. H., 1994 - Composición, abundancia y riqueza especifica de la ictiofauna de Bahía concepción, Baja California sur, México, Ciencias Marinas, 20, 3, 321-350.

81. Rodríguez-Zaragoza F. A., Cupul-Magaña A. L., Galván-Villa C. M., Ríos-Jara E., Ortiz M., Robles-Jarero, E. G. and Arias-González J. E., 2011 - Additive partitioning of reef fish diversity variation, a promising marine biodiversity management tool, Biodiversity and Conservation, 20, 8, 1655-1675, DOI: 10.1007/s10531-011-0053-9.

82. Rouphael A. B. and Inglis G. J., 2000 - Take only photographs and leave only footprints? An experimental study of the impacts of underwater photographers on coral reef dive sites, Biological Conservation, 100, 281-287.

83. Santos T. and Tellería J. L. 2006 - Pérdida y fragmentación del hábitat: efecto sobre la conservación de las especies, Ecosistemas, 15, 2, 3-12. (in Spanish)

84. Steneck R. S., Arnold S. N. and Mumby P. J., 2014 - Experiment mimics fihing on parrotfih: insights on coral reef recovery and alternative attractors, Marine Ecology Progress Series, 506, 115-127, https://doi.org/10.3354/meps10764.

85. Sonco S. R., 2013 - Estudio de la diversidad alfa y beta en tres localidades de un bosque montano en la región de Madidi, La Paz Bolivia, Tesis de grado, Facultad y Carrera de Ingeniera Agrónoma, Universidad Mayor de San Andrés, 1-154. (in Spanish)

86. Southward A. J. and Boalch G. T., 1994 - The effect of changing climate on marine life: past events and future predictions, Exeter Maritime Studies, 9, 101-143. (in Spanish)

87. Southward A. J., Hawkins S. J. and Burrows M. T., 1995 - Seventy years observations in distribution and abundance of zooplankton and intertidal organisms in the western English Channel in relation to rising sea temperature, Journal of Thermal Biology, 20, 127-155.

88. Stehli F. G., Douglas R. G. and Nowell N. D., 1969 - Generation and maintenance of gradients in taxonomic diversity, Science, 164, 947-949.

89. Stevens G. C., 1989 - The latitudinal gradient in geographical range: how so many species coexist in the tropics, American Naturalist, 133, 240-430.

90. Vega A., Zayas I., Zayas C., Fernández A. and Peña C., 2004 - Sea shell of Yuraguanal beach, Holguin province, Cuba, Of Sea and Shore, 26, 1, 4-9.

91. Valdivia A., Cox C. E. and Bruno J. F., 2017 - Predatory fish depletion and recovery potential on Caribbean reefs, Sciences Advanced, 3, 160, 1303, https://doi.org/10.1126/sciadv.1601303.

92. Williams I. D. and Polunin N. V. C., 2001 - Large scale associations between macro algal cover and grazer biomass on mid-depth reefs in the Caribbean, Coral Reefs, 19, 358-366.

93. Whittaker R. H., 1972 - Evolution and measurement of species diversity, Taxon, 21, 2/3, 213251.

94. Zakai D. and Chadwick-Furman N. E., 2002 - Impacts of intensive recreational diving on reef corals at Eilat, northern Red Sea, Biology Conservation, 105, 179-187. 